\title{
Collective Modes in Neutrino 'Beam' Electron-Positron Plasma Interactions
}

\author{
Hans-Thomas Elze ${ }^{1}$, Takeshi Kodama ${ }^{1}$ and Reuven Opher ${ }^{2}$ \\ ${ }^{1}$ Universidade Federal do Rio de Janeiro, Instituto de Física \\ Caixa Postal 68.528, 21945-970 Rio de Janeiro, RJ, Brazil \\ and \\ ${ }^{2}$ Instituto Astronômico e Geofísico, USP \\ Caixa Postal 9638, 01065-970, São Paulo, SP, Brazil
}

June 2000

\begin{abstract}
We derive semiclassical neutrino-electron transport equations in the collisionless (Vlasov) limit from the coupled Dirac equations, incorporating the charged and neutral weak current-current as well as electromagnetic interactions. A corresponding linear response theory is derived. In particular, we calculate the response functions for a variety of beam-plasma geometries, which are of interest in a supernova scenario. We apply this to the study of plasmons and to a new class of collective pharon resonance modes, which are characterized by $\omega<q$. We find that the growth rates of the unstable modes correspond to a strongly temperature $\left(\propto T_{\nu}^{2} T_{e}^{3}\right)$ and linearly momentum dependent e-folding length of about $10^{10} \mathrm{~km}$ under typical conditions for Type II supernovae. This appears to rule out such long-wavelength collective modes as an efficient means of depositing neutrino energy into the plasma sphere.

PACS numbers: 05.60.-k, 13.15.+g, 14.60.Lm, 97.60.Bw
\end{abstract}

\section{Introduction}

Neutrino transport processes are known to play a major role in the energy-momentum flow powering the dynamics of Type II supernovae [1, 2, 3]. Generally, it has been that the collision-dominated aspects have been studied in detail, leading to substantial progress in the understanding of these stellar explosions, while, however, still leaving open some problems in the quantitative description of their spatio-temporal (hydrodynamic) evolution.

An earlier work by Bethe [何, which introduced the idea of a modified in-medium neutrino dispersion relation and of a corresponding effective Hamiltonian, was used in the series of papers [5] to describe the collective interaction of an intense neutrino flux (from the supernova core) with an electron-positron plasma (the supernova atmosphere) of comparatively low temperature. Their tentative conclusion was that a particular induced plasma instability may be much more efficient than traditional collision dominated mechanisms, i.e., faster by many orders of magnitude, in depositing the neutrino energy into the plasma sphere [5].

However, the approach in these papers is subject to criticism, since there is no physical or formal justification (such as a hypothetical condensate) for the scalar 'bosonic' collective neutrino wave function used. In particular, the implied quantum phase coherence of the neutrinos appears hard to justify. 
Considering their incoherent thermal production and the effective duration or length of the 'beam', no bunching effects are to be expected. Moreover, it is somewhat hidden in their phaenomenological approach how the precise V-A tensor structure of the electroweak current-current interactions can be taken into account. This has also been pointed out in Ref. [6] recently. Employing finite temperature field theory, Bento studies the excitation/damping of longitudinal electromagnetic plasmons (Langmuir modes) in the electron plasma under the influence of a neutrino flux. His results indicate that this type of collective mode instabilities "... do not seem to be a viable mechanism of substantial energy transfer from neutrinos to a supernova plasma" [6]. We confirm this.

It is the purpose of our present work to systematically derive the transport equations for the neutrino-electron system from first principles (Section 2), as well as the relevant dispersion relations (Section 3). We introduce appropriate spinor Wigner functions, while deriving the detailed chiral structure of the neutrino Wigner function in the Appendix. Previously, only the phenomenological approach [5] or the perturbative finite-temperature field theory [6, 6] were applied. Our general derivations may also prove useful for other astrophysical applications, such as those involving strong magnetic fields or, generally, neutrino transport under mean field conditions.

In the collisionless regime, the results of Sections 2 and 3 allow us to investigate, in detail, the collective modes in the highly anisotropic neutrino 'beam' plus electron-positron plasma system. We find longitudinal and transverse plasmons, which are only perturbatively modified by the neutrino flux.

Furthermore, we also find a new class of growing, as well as decaying collective oscillations, nonexistent in isotropic equilibrium plasmas, which we name 'pharons 7 . They are caused by a resonance effect, generally at a frequency $\omega$ less than the momentum $q$, due to the unbalanced neutrino momentum distribution, which is characterized by a finite opening angle with respect to the beam axis. We study such modes with the wave vector parallel to the beam direction (Type I pharons), as well as with the wave vector orthogonal to the beam direction (Type II pharons).

Geometrically, the Type II situation corresponds most closely to the one where the two-stream instabilities would be expected to occur in ordinary plasmas. We investigate whether such instabilities are induced by the weak current-current interactions and, depending on the growth rates, may provide an essential contribution to the still partly elusive energy transfer mechanism in Type II supernovae (Section 3).

The collective two-stream filamentation instability is well known to occur in ordinary plasmas due to the electromagnetic Lorentz force [8, 9]. More recently, it has also been studied in the context of strong (color-electromagnetic) interactions, where two interpenetrating parton beams describe highenergy nuclear reactions, see [10] and further references therein.

More generally, one may expect such 'hydrodynamic' instabilities in interacting many-body systems, in particular, in plasmas with interactions mediated by the Standard Model gauge fields, whenever the system consists of two or more components with considerably different momentum space distributions [8, 9, 10]. In these cases, perturbations which, loosely speaking, are transverse to a predominant collective flow, tend to be amplified by the collective feedback effect of the effective long-range forces of mean field type.

Consequently, we are motivated in this study by the supernova geometry, where the radially outward streaming neutrinos interact with the electron-positron plasma, which may produce a variety of collective instabilities.

\footnotetext{
${ }^{1}$ After the island of Pharos, where the famous lighthouse of ancient Alexandria was constructed under the order of Ptolemeus II.
} 


\section{The Coupled Neutrino-Electron Transport Equations}

Our derivation of mean field transport equations will follow the successful strategy developed earlier for QED [11], QCD [12], and hadronic matter [13]. The basic idea can be easily summarized as follows: Starting from the underlying field operator equations of the model under consideration, one converts these into corresponding Wigner operator equations, i.e. for the density operator in the Wigner representation. In the appropriate mean field approximation the latter can be converted into a closed set of Wigner function equations (cf. Section 2.1); furthermore, performing a consistent $\hbar$-expansion, the most relevant semiclassical (Vlasov type) transport equations for the coupled relativistic phase space distributions are obtained (cf. Section 2.2). - Presently, our notation and conventions follow those of Ref. 11.

\subsection{From Dirac's to Mean Field Quantum Transport Equations}

We add the effective local coupling terms,

$$
\mathcal{L}_{\text {int }} \equiv-\frac{G_{F}}{\sqrt{2}}\left[\bar{\psi}^{(\nu)} \gamma_{\mu}\left(1-\gamma_{5}\right) \psi^{(\nu)}\right]\left[\bar{\psi}^{(e)} \gamma^{\mu}\left(c_{V}-c_{A} \gamma_{5}\right) \psi^{(e)}\right]
$$

to the free Lagrangian densities of the electrons and electron neutrinos (including their antiparticles). They represent the weak charged and neutral current-current interactions of the Standard Model in the appropriate low-energy limit, with $c_{V}=\frac{1}{2}+2 \sin ^{2} \theta_{W}$ and $c_{A}=\frac{1}{2}$, using standard notation [6]. For $\mu$ - and $\tau$-neutrinos, with only the neutral current interaction contributing, $c_{V, A} \rightarrow c_{V, A}-1$. Here the neutrinos are described by four-component spinors as well and we allow for a small but finite neutrino mass, taking the growing evidence into account [14]. Eventually, however, we will pass to the massless limit, since for our applications the masses of order eV are definitely negligible compared to the relevant energy scales of order $\mathrm{MeV}$.

The electromagnetic interaction of the electrons must be added to the interaction of Eq. (11). For the derivation of the transport equations, however, this does not introduce a new element. The corresponding modifications will be added at the end of this section, making use of the earlier QED results [1].

The resulting Dirac (Heisenberg operator) equations for the electrons and neutrinos, incorporating the interaction (11) in the mean field (Hartree) approximation, can be written in the form:

$$
\left\{i \gamma \cdot \partial_{x}-m^{(l)}-J^{\left(l^{\prime}\right)} \cdot \gamma\left(c_{V}^{(l)}-c_{A}^{(l)} \gamma_{5}\right)\right\} \psi^{(l)}=0
$$

where $l=e, \nu$ denotes the electron and neutrino case, respectively, and $c_{V, A}^{(e)} \equiv c_{V, A}, c_{V, A}^{(\nu)} \equiv 1$; the neutrino current has to be inserted into the electron equation and vice versa, as indicated by $J^{\left(l^{\prime}\right)}$ here. The V-A four-currents are defined by:

$$
J_{\mu}^{(l)} \equiv \frac{G_{F}}{\sqrt{2}}\left\langle: \bar{\psi}^{(l)} \gamma_{\mu}\left(c_{V}^{(l)}-c_{A}^{(l)} \gamma_{5}\right) \psi^{(l)}:\right\rangle
$$

where the expectation value of the normal-ordered product refers to the ensemble characterizing the state of the system, which will be specified in more detail later.

Introducing the Wigner functions, i.e. (4x4)-matrices with respect to the spinor indices which depend on space-time and four-momentum coordinates [11]:

$$
W_{\alpha \beta}^{(l)}(x, p) \equiv \int \frac{\mathrm{d}^{4} y}{(2 \pi \hbar)^{4}} \mathrm{e}^{-i p \cdot y / \hbar}\left\langle: \bar{\psi}_{\beta}^{(l)}(x+y / 2) \psi_{\alpha}^{(l)}(x-y / 2):\right\rangle
$$


and with the $\hbar$-dependence made explicit, the currents of Eq. (3) can be expressed as:

$$
J_{\mu}^{(l)}(x)=\frac{G_{F}}{\sqrt{2}} \operatorname{tr} \int \mathrm{d}^{4} p \gamma_{\mu}\left(c_{V}^{(l)}-c_{A}^{(l)} \gamma_{5}\right) W^{(l)}(x, p)
$$

with the trace refering to the spinor indices.

Multiplying the Dirac equations (2) with the respective adjoint spinor and making use of Eqs. (3)(5), they can be converted to the Wigner representation. This yields the coupled electron and neutrino quantum transport equations:

$$
\left(\gamma \cdot K-m^{(l)}\right) W^{(l)}(x, p)=\exp \left(-\frac{i \hbar}{2} \partial_{x} \cdot \partial_{p}\right) J^{\left(l^{\prime}\right)}(x) \cdot \gamma\left(c_{V}^{(l)}-c_{A}^{(l)} \gamma_{5}\right) W^{(l)}(x, p),
$$

where $K_{\mu} \equiv p_{\mu}+\frac{i \hbar}{2} \partial_{x^{\mu}}$ and the partial derivative with respect to $x$ on the right-hand side acts only on the current $J^{\left(l^{\prime}\right)}$. If it were not for the $\mathrm{V}$-A factor on the right-hand side, the structure of these equations would be analogous to Eq. (8) of Ref. [13] and could be analyzed accordingly.

In order to proceed here, we employ the decomposition of the spinor Wigner functions [11]:

$$
W^{(l)}=\mathcal{F}^{(l)}+i \gamma^{5} \mathcal{P}^{(l)}+\gamma^{\mu} \mathcal{V}_{\mu}^{(l)}+\gamma^{\mu} \gamma^{5} \mathcal{A}_{\mu}^{(l)}+\frac{1}{2} \sigma^{\mu \nu} \mathcal{S}_{\mu \nu}^{(l)}
$$

i.e., in terms of scalar, pseudoscalar, vector, axial vector, and antisymmetric tensor components:

$$
\begin{aligned}
\mathcal{F}^{(l)}(x, p) & \equiv \frac{1}{4} \operatorname{tr} W^{(l)}(x, p) \\
\mathcal{P}^{(l)}(x, p) & \equiv-\frac{1}{4} i \operatorname{tr} \gamma^{5} W^{(l)}(x, p) \\
\mathcal{V}_{\mu}^{(l)}(x, p) & \equiv \frac{1}{4} \operatorname{tr} \gamma_{\mu} W^{(l)}(x, p), \\
\mathcal{A}_{\mu}^{(l)}(x, p) & \equiv \frac{1}{4} \operatorname{tr} \gamma_{5} \gamma_{\mu} W^{(l)}(x, p) \\
\mathcal{S}_{\mu \nu}^{(l)}(x, p) & \equiv \frac{1}{4} \operatorname{tr} \sigma_{\mu \nu} W^{(l)}(x, p),
\end{aligned}
$$

which are real functions. Thus, for example, we obtain:

$$
J_{\mu}^{(l)}(x)=4 \frac{G_{F}}{\sqrt{2}} \int \mathrm{d}^{4} p\left[c_{V}^{(l)} \mathcal{V}_{\mu}^{(l)}(x, p)+c_{A}^{(l)} \mathcal{A}_{\mu}^{(l)}(x, p)\right],
$$

using Eqs. (5) and (10), (11). Only these (axial) vector currents couple the transport equations (6).

We introduce an abbreviation for the shift operator appearing in Eqs. (6),

$$
\mathcal{J}_{\mu}^{(l)} \equiv \exp \left(-\frac{i \hbar}{2} \partial_{x} \cdot \partial_{p}\right) J_{\mu}^{(l)}(x)=\left[\cos \left(\frac{\hbar}{2} \partial_{x} \cdot \partial_{p}\right)-i \sin \left(\frac{\hbar}{2} \partial_{x} \cdot \partial_{p}\right)\right] J_{\mu}^{(l)}(x) \equiv \mathcal{R}_{\mu}^{(l)}-i \mathcal{I}_{\mu}^{(l)}
$$

where $\partial_{x}$ acts only on the current $J_{\mu}^{(l)}$, as before. Then, making use of the commutation and trace relations of the $\gamma$-matrices, we decompose Eqs. (6) in terms of the Wigner function components, Eqs. (8)-(12). Thus we obtain the set of coupled equations:

$$
\begin{aligned}
K \cdot \mathcal{V}^{(l)}-m^{(l)} \mathcal{F}^{(l)} & =\mathcal{J}^{\left(l^{\prime}\right)} \cdot\left(c_{V}^{(l)} \mathcal{V}^{(l)}+c_{A}^{(l)} \mathcal{A}^{(l)}\right), \\
i K \cdot \mathcal{A}^{(l)}+m^{(l)} \mathcal{P}^{(l)} & =i \mathcal{J}^{\left(l^{\prime}\right)} \cdot\left(c_{V}^{(l)} \mathcal{A}^{(l)}+c_{A}^{(l)} \mathcal{V}^{(l)}\right),
\end{aligned}
$$




$$
\begin{gathered}
K_{\mu} \mathcal{F}^{(l)}-i K^{\nu} \mathcal{S}_{\mu \nu}^{(l)}-m^{(l)} \mathcal{V}_{\mu}^{(l)} \\
=\mathcal{J}^{\left(l^{\prime}\right) \lambda}\left(g_{\mu \lambda}\left(c_{V}^{(l)} \mathcal{F}^{(l)}-i c_{A}^{(l)} \mathcal{P}^{(l)}\right)-i c_{V}^{(l)} \mathcal{S}_{\mu \lambda}^{(l)}-\frac{1}{2} c_{A}^{(l)} \epsilon_{\mu \lambda \nu \nu^{\prime}} \mathcal{S}^{(l) \nu \nu^{\prime}}\right), \\
i K_{\mu} \mathcal{P}^{(l)}+\frac{1}{2} \epsilon_{\mu \lambda \nu \nu^{\prime}} K^{\lambda} \mathcal{S}^{(l) \nu \nu^{\prime}}-m^{(l)} \mathcal{A}_{\mu}^{(l)} \\
=\mathcal{J}^{\left(l^{\prime}\right) \lambda}\left(g_{\mu \lambda}\left(i c_{V}^{(l)} \mathcal{P}^{(l)}-c_{A}^{(l)} \mathcal{F}^{(l)}\right)+\frac{1}{2} c_{V}^{(l)} \epsilon_{\mu \lambda \nu \nu^{\prime}} \mathcal{S}^{(l) \nu \nu^{\prime}}+i c_{A}^{(l)} \mathcal{S}_{\mu \lambda}^{(l)}\right), \\
i\left(K_{\mu} \mathcal{V}_{\nu}^{(l)}-K_{\nu} \mathcal{V}_{\mu}^{(l)}\right)-\epsilon_{\mu \nu \lambda \nu^{\prime}} K^{\lambda} \mathcal{A}^{l \nu^{\prime}}+m^{(l)} \mathcal{S}_{\mu \nu}^{(l)}= \\
\mathcal{J}^{\left(l^{\prime}\right) \lambda}\left(i\left[g_{\mu \lambda}\left(c_{V}^{(l)} \mathcal{V}^{(l)}+c_{A}^{(l)} \mathcal{A}^{(l)}\right)_{\nu}-\ldots \mu \leftrightarrow \nu\right]\right. \\
\left.-\epsilon_{\mu \nu \lambda \nu^{\prime}}\left(c_{V}^{(l)} \mathcal{A}^{(l)}+c_{A}^{(l)} \mathcal{V}^{(l)}\right)^{\nu^{\prime}}\right),
\end{gathered}
$$

with $K_{\mu}$ as defined after Eq. (6).

As is well known from other cases [11, 12, 13, the real and imaginary parts of these coupled equations can be separated and eventually will thus lead to the proper transport equations in phase space and the generalizations of the mass-shell constraint, cf. Section 2.2 .

Furthermore, we observe that the left-hand sides of Eqs. (15)-(19) formally coincide with Eqs. (5.7)(5.11) of Ref. [11]. There, however, the corresponding operator $K^{\mu}$ for electrically charged particles necessarily incorporates the effects of the Lorentz force in the external field (Hartree) approximation.

Due to the linearity of the Dirac equation with respect to the weak and electromagnetic interaction terms, i.e. with the derivative in Eq. (2) replaced according to the minimal coupling rule, $\partial_{x}^{\mu} \rightarrow$ $\partial_{x}^{\mu}+i e A^{\mu}(x)$, it is straighforward to incorporate the electromagnetic interaction into Eqs. (15)-(19) for the electron-positron case. Making use of the earlier QED results, this is achieved by the substitution:

$$
\begin{aligned}
K^{\mu} & \equiv \Pi^{\mu}+\frac{i \hbar}{2} \nabla^{\mu}, \\
\nabla^{\mu} & \equiv \partial_{x}^{\mu}-e j_{0}\left(\frac{\hbar}{2} \partial_{x} \cdot \partial_{p}\right) F^{\mu \nu} \partial_{p^{\nu}}, \\
\Pi^{\mu} & \equiv p^{\mu}-e \frac{\hbar}{2} j_{1}\left(\frac{\hbar}{2} \partial_{x} \cdot \partial_{p}\right) F^{\mu \nu} \partial_{p^{\nu}},
\end{aligned}
$$

where $j_{0}$ and $j_{1}$ are the conventional spherical Bessel functions, cf. Eqs. (4.19)-(4.21) of Ref. [11]; the derivatives $\partial_{x}$ in their arguments act only on the electromagnetic field strength tensor entering here, $F^{\mu \nu}(x) \equiv \partial_{x}^{\mu} A^{\nu}(x)-\partial_{x}^{\nu} A^{\mu}(x)$. Our convention is that $e$ denotes the electron charge.

With the electromagnetic fields incorporated, we also need to include the Maxwell equation,

$$
\partial_{\mu} F^{\mu \nu}(x)=J_{\mathrm{em}}^{\nu}(x) \equiv e \operatorname{tr} \int \mathrm{d}^{4} p \gamma^{\nu} W^{(e)}(x, p)
$$

which consistently determines $F^{\mu \nu}$ in terms of the electromagnetic four-current $J_{\mathrm{em}}^{\nu}$. However, an important remark is in order here. Together with Eqs. (20)-(22) also the definition of the Wigner function (四) has to be modified. In order to preserve the gauge covariance of the equations, one has to include an appropriate electromagnetic phase factor ('Schwinger string') [11, 12]. Since it will not appear explicitly in any of our further derivations or applications, it may presently suffice to keep this in mind.

This completes the derivation of the coupled transport equations for a system of electrons, neutrinos, and electromagnetic fields in accordance with the Standard Model and in the collisionless (Vlasov) limit. 


\subsection{The Semiclassical Limit}

Our aim in this section is to extract the relevant semiclassical equations from the quantum transport equations which we obtained in the previous section, Eqs. (15)-(19) in particular. Taking the explicit $\hbar$-dependence into account, which enters through the definitions of the shift and kinetic operators in Eqs. (14) and (20)-(22) respectively, it becomes obvious how to expand the equations in powers of $\hbar$. Since the leading terms of the real and imaginary parts of the equations start out with different powers, it is useful to separate them, similarly to what was previously done [11, 12, 13].

Furthermore, we presently simplify the set of equations by assuming a spin saturated electronpositron plasma, i.e. without the spin polarization effects which may be induced by strong magnetic fields, for example. Thus, for the $e^{+} e^{-}$plasma, we have no pseudoscalar or axial vector densities, cf. Eqs. (7)-(12).

Also, the Standard Model neutrino-antineutrino system consists strictly only of left-handed neutrinos $\nu_{L}$ and a right-handed antineutrinos $\bar{\nu}_{R}$, if we appropriately neglect here their tiny (possibly finite) masses. In this case, as we show in the Appendix, only the equal vector and axial vector densities contribute to the neutrino Wigner function, while all other densities vanish in the massless limit.

These approximations serve as a working hypothesis for our study of the collective modes and their (in)stability in a supernova environment in Section 3. Eventually, however, the analysis of the complete coupled set of equations (15)-(19) and (23) should be performed, considering the presence or generation of strong magnetic fields during supernova explosions or other astrophysical processes [15, 16, 17] (and references therein).

\subsubsection{The Semiclassical $e^{+} e^{-}$Transport Equations}

Implementing $\mathcal{P}^{(e)}=\mathcal{A}_{\mu}^{(e)} \equiv 0$ (spin saturation) and separating the real and imaginary parts of Eqs. (15)-(19) with the help of Eqs. (14) and (20), we obtain for the $e^{+} e^{-}$plasma the set of equations:

$$
\begin{aligned}
& \Pi \cdot \mathcal{V}^{(e)}-m^{(e)} \mathcal{F}^{(e)}=0 \\
& \hbar \nabla \cdot \mathcal{V}^{(e)}=0 \\
& 0=\mathcal{R}^{(\nu)} \cdot \mathcal{V}^{(e)} \\
& 0=\mathcal{I}^{(\nu)} \cdot \mathcal{V}^{(e)}, \\
& \Pi_{\mu} \mathcal{F}^{(e)}+\frac{\hbar}{2} \nabla^{\nu} \mathcal{S}_{\mu \nu}^{(e)}-m^{(e)} \mathcal{V}_{\mu}^{(e)}=\mathcal{R}^{(\nu) \lambda}\left(g_{\mu \lambda} c_{V} \mathcal{F}^{(e)}-\frac{1}{2} c_{A} \epsilon_{\mu \lambda \nu \nu^{\prime}} \mathcal{S}^{(e) \nu \nu^{\prime}}\right)-c_{V} \mathcal{I}^{(\nu) \lambda} \mathcal{S}_{\mu \lambda}^{(e)} \\
& \frac{\hbar}{2} \nabla_{\mu} \mathcal{F}^{(e)}-\Pi^{\nu} \mathcal{S}_{\mu \nu}^{(e)}=-\mathcal{I}^{(\nu) \lambda}\left(g_{\mu \lambda} c_{V} \mathcal{F}^{(e)}-\frac{1}{2} c_{A} \epsilon_{\mu \lambda \nu \nu^{\prime}} \mathcal{S}^{(e) \nu \nu^{\prime}}\right)-c_{V} \mathcal{R}^{(\nu) \lambda} \mathcal{S}_{\mu \lambda}^{(e)} \\
& \frac{1}{2} \epsilon_{\mu \lambda \nu \nu^{\prime}} \Pi^{\lambda} \mathcal{S}^{(e) \nu \nu^{\prime}}=-\mathcal{R}^{(\nu) \lambda}\left(g_{\mu \lambda} c_{A} \mathcal{F}^{(e)}-\frac{1}{2} c_{V} \epsilon_{\mu \lambda \nu \nu^{\prime}} \mathcal{S}^{(e) \nu \nu^{\prime}}\right)+c_{A} \mathcal{I}^{(\nu) \lambda} \mathcal{S}_{\mu \lambda}^{(e)}, \\
& \frac{\hbar}{4} \epsilon_{\mu \lambda \nu \nu^{\prime}} \nabla^{\lambda} \mathcal{S}^{(e) \nu \nu^{\prime}}=\mathcal{I}^{(\nu) \lambda}\left(g_{\mu \lambda} c_{A} \mathcal{F}^{(e)}-\frac{1}{2} c_{V} \epsilon_{\mu \lambda \nu \nu^{\prime}} \mathcal{S}^{(e) \nu \nu^{\prime}}\right)+c_{A} \mathcal{R}^{(\nu) \lambda} \mathcal{S}_{\mu \lambda}^{(e)} \\
& -\frac{\hbar}{2}\left(\nabla_{\mu} \mathcal{V}_{\nu}^{(e)}-\nabla_{\nu} \mathcal{V}_{\mu}^{(e)}\right)+m^{(e)} \mathcal{S}_{\mu \nu}^{(e)}=c_{V}\left(\mathcal{I}_{\mu}^{(\nu)} \mathcal{V}_{\nu}^{(e)}-\mathcal{I}_{\nu}^{(\nu)} \mathcal{V}_{\mu}^{(e)}\right)-c_{A} \epsilon_{\mu \nu \lambda \nu^{\prime}} \mathcal{R}^{(\nu) \lambda} \mathcal{V}^{(e) \nu^{\prime}} \\
& \Pi_{\mu} \mathcal{V}_{\nu}^{(e)}-\Pi_{\nu} \mathcal{V}_{\mu}^{(e)}=c_{V}\left(\mathcal{R}_{\mu}^{(\nu)} \mathcal{V}_{\nu}^{(e)}-\mathcal{R}_{\nu}^{(\nu)} \mathcal{V}_{\mu}^{(e)}\right)+c_{A} \epsilon_{\mu \nu \lambda \nu^{\prime}} \mathcal{I}^{(\nu) \lambda} \mathcal{V}^{(e) \nu^{\prime}}
\end{aligned}
$$


where the constraints (26) and (27), which resulted from Eq. (16), where taken into account in Eqs. (24) and (25), which resulted from Eq. (15); we used $c_{V, A}^{(e)}=c_{V, A}$, cf. Eqs. (1), (2)).

We proceed to evaluate the limit $\hbar \rightarrow 0$ of the above system of equations. To begin with, we obtain from Eqs. (14) that $\mathcal{R}_{\mu}^{(l)}=J_{\mu}^{(l)}+O\left(\hbar^{2}\right)$ and $\mathcal{I}_{\mu}^{(l)}=\frac{\hbar}{2} \partial_{x} \cdot \partial_{p} J_{\mu}^{(l)}+O\left(\hbar^{3}\right)$ and from Eqs. (21), (22) that $\Pi^{\mu}=p^{\mu}+O\left(\hbar^{2}\right)$ and $\nabla^{\mu}=\partial_{x}^{\mu}-e F^{\mu \nu} \partial_{p^{\nu}}+O\left(\hbar^{2}\right)$.

Then, first of all, the vector density can formally be calculated from Eq. (28):

$$
\begin{aligned}
\mathcal{V}_{\mu}^{(e)=} \frac{1}{m^{(e)}} & \left(\left(p_{\mu}-c_{V} J_{\mu}^{(\nu)}\right) \mathcal{F}^{(e)}+\frac{1}{2} c_{A} \epsilon_{\mu \lambda \nu \nu^{\prime}} J^{(\nu) \lambda} \mathcal{S}^{l \nu \nu^{\prime}}\right. \\
& \left.+\frac{\hbar}{2}\left(\partial_{x}^{\nu}-e F^{\nu \lambda} \partial_{p^{\lambda}}\right) \mathcal{S}_{\mu \nu}^{(e)}+c_{V} \frac{\hbar}{2} \partial_{x} \cdot \partial_{p} J^{(\nu) \lambda} \mathcal{S}_{\mu \lambda}^{(e)}\right)+O\left(\hbar^{2}\right)
\end{aligned}
$$

where the right-hand side is to be evaluated consistently to first order; we recall that $\partial_{x}$ acts only on $J^{(\nu)}$ in the last term. Similarly, we obtain from Eq. (32):

$$
\begin{aligned}
\mathcal{S}_{\mu \nu}^{(e)}= & \frac{1}{m^{(e)}}\left(-c_{A} \epsilon_{\mu \nu \lambda \nu^{\prime}} J^{(\nu) \lambda} \mathcal{V}^{(e) \nu^{\prime}}\right. \\
& \left.+\frac{\hbar}{2}\left[\left(\partial_{x^{\mu}}-e F_{\mu \lambda} \partial_{p}^{\lambda}\right) \mathcal{V}_{\nu}^{(e)}-\ldots \mu \leftrightarrow \nu\right]+c_{V} \frac{\hbar}{2} \partial_{x} \cdot \partial_{p}\left(J_{\mu}^{(\nu)} \mathcal{V}_{\nu}^{(e)}-J_{\nu}^{(\nu)} \mathcal{V}_{\mu}^{(e)}\right)\right)+O\left(\hbar^{2}\right)
\end{aligned}
$$

with a contribution at $O\left(\hbar^{0}\right)$ in the absence of a pseudo-vector (or -scalar) density, in distinction to the QED case of Ref. 11]. Taking the limit $\hbar \rightarrow 0$, we solve Eqs. (34)-(35) in terms of the scalar density $\mathcal{F}^{(e)}$ or, rather, the modified scalar density,

$$
\tilde{f}^{(e)}(x, p) \equiv \frac{\mathcal{F}^{(e)}(x, p)}{1+\left(c_{A} / m^{(e)}\right)^{2} J^{(\nu)}(x) \cdot J^{(\nu)}(x)} .
$$

The results are:

$$
\begin{aligned}
\mathcal{V}_{\mu}^{(e)} & =\frac{1}{m^{(e)}}\left(p_{\mu}-c_{V} J_{\mu}^{(\nu)}\right) \tilde{f}^{(e)}, \\
\mathcal{S}_{\mu \nu}^{(e)} & =\frac{c_{A}}{m^{(e) 2}} \epsilon_{\mu \nu \nu^{\prime} \lambda}\left(p^{\nu^{\prime}}-c_{V} J^{(\nu) \nu^{\prime}}\right) J^{(\nu) \lambda} \tilde{f}^{(e)},
\end{aligned}
$$

where we made use of the constraint $(26)$, i.e. $J^{(\nu)} \cdot \mathcal{V}=0$ for $\hbar \rightarrow 0$, and conveniently added a term on the right-hand side of Eq. (38) which vanishes identically. Thus, we find that in the semiclassical limit the spinor Wigner functions for the spin saturated system are completely determined by the scalar density, cf. Eq. (7).

Next, using Eqs. (36) and (37), the Eq. (25) yields a transport equation for the scalar density:

$$
\left(\partial_{x}^{\mu}-e F^{\mu \nu} \partial_{p^{\nu}}\right)\left(p_{\mu}-c_{V} J_{\mu}^{(\nu)}\right) \tilde{f}^{(e)}=\left(\left(p_{\mu}-c_{V} J_{\mu}^{(\nu)}\right)\left(\partial_{x}^{\mu}-e F^{\mu \nu} \partial_{p^{\nu}}\right)-c_{V}\left(\partial_{x} \cdot J^{(\nu)}\right)\right) \tilde{f}^{(e)}=0,
$$

i.e. in the limit $\hbar \rightarrow 0$. Similarly, we obtain from Eq. (24) together with Eq. (37) a constraint equation:

$$
\left(\left(p-c_{V} J^{(\nu)}\right)^{2}-m^{(e) 2}\left(1+\left(c_{A} / m^{(e)}\right)^{2} J^{(\nu)} \cdot J^{(\nu)}\right)\right) \tilde{f}^{(e)}=0
$$

where we also used the constraint (26) in the form:

$$
\left(p-c_{V} J^{(\nu)}\right) \cdot J^{(\nu)} \tilde{f}^{(e)}=0
$$

which is appropriate in this limit. 
Clearly, the Eq. (40) demonstrates that it is the kinetic momentum,

$$
k_{\mu}^{(e)} \equiv p_{\mu}-c_{V} J_{\mu}^{(\nu)}(x)
$$

which should be related to a classical mass-shell constraint. Therefore, we redefine the scalar density as a function of the kinetic momentum $k$,

$$
\tilde{f}^{(e)}(x, p)=\tilde{f}^{(e)}\left(x, k^{(e)}+c_{V} J^{(\nu)}\right) \equiv f^{(e)}(x, k),
$$

instead of the canonical momentum $p$; we will omit the superscript from $k^{(e)}$, since it is identical to the one of $f^{(e)}$ in the respective equations. This implies:

$$
\left.\partial_{x}^{\mu} \tilde{f}^{(e)}\right|_{p}=\left.\partial_{x}^{\mu} f^{(e)}\right|_{k}-c_{V}\left(\partial_{x}^{\mu} J_{\nu}^{(\nu)}\right) \partial_{k}^{\nu} f^{(e)}
$$

For the redefined variable and scalar density function, the $e^{+} e^{-}$mass-shell constraint follows:

$$
\left.\left(k^{2}-m^{(e) 2}-c_{A}^{2} J^{(\nu)} \cdot J^{(\nu)}\right)\right) f^{(e)}=0
$$

instead of Eq. (40). Furthermore, we finally obtain from Eq. (39) the Vlasov type transport equation for the scalar $e^{+} e^{-}$density:

$$
\begin{aligned}
& \left(k \cdot \partial_{x}-c_{V} k_{\mu}\left(\partial_{x}^{\mu} J^{(\nu) \nu}\right) \partial_{k^{\nu}}-c_{V}\left(\partial_{x} \cdot J^{(\nu)}\right)-e k_{\mu} F^{\mu \nu} \partial_{k^{\nu}}\right) f^{(e)} \\
& \left.\quad=\left(k \cdot \partial_{x}-k_{\mu}\left(c_{V}\left[\partial_{x}^{\mu} J^{(\nu) \nu}-\partial_{x}^{\nu} J^{(\nu) \mu}\right]+e F^{\mu \nu}\right) \partial_{k^{\nu}}\right)\right) f^{(e)}=0
\end{aligned}
$$

rewriting and using here the appropriate leading order in $\hbar$ form of the constraint (27):

$$
\left(\partial_{x}^{\nu} J^{(\nu) \mu}\right) \partial_{p^{\nu}}\left(p_{\mu}-c_{V} J_{\mu}^{(\nu)}\right) \tilde{f}^{(e)}=\left(k_{\mu}\left(\partial_{x}^{\nu} J^{(\nu) \mu}\right) \partial_{k^{\nu}}+\left(\partial_{x} \cdot J^{(\nu)}\right)\right) f^{(e)}=0
$$

In particular, we also employed Eq. (37), rewritten now simply as:

$$
\mathcal{V}_{\mu}^{(e)}=\frac{k_{\mu}}{m^{(e)}} f^{(e)}
$$

We observe that the weak current-current interaction leads to an antisymmetric tensor coupling in the transport equation (46), which is analogous to the electromagnetic field strength coupling.

Furthermore, we remark that there are remaining equations of the set (24)-(33) which we did not consider here, since the dynamics can be represented completely in terms of the scalar density $\mathcal{F}^{(e)}$, recall Eqs. (36)-(38). Similarly as in the QED case of Ref. [11], they could be shown to be satisfied identically to leading order in the $\hbar$-expansion, which we do not pursue here.

\subsubsection{The Semiclassical $\nu_{L} \bar{\nu}_{R}$ Transport Equations and Currents}

For approximately massless neutrinos, with $\mathcal{V}_{\mu}^{(\nu)}=\mathcal{A}_{\mu}^{(\nu)}$ and $\mathcal{F}=\mathcal{P}=\mathcal{S}_{\mu \nu} \equiv 0$ (see Appendix), we obtain a much simpler set of equations from Eqs. (15)-(19):

$$
\begin{aligned}
\left(K-2 \mathcal{J}^{(e)}\right) \cdot \mathcal{V}^{(\nu)} & =0, \\
i\left[\left(K-2 \mathcal{J}^{(e)}\right)_{\mu} \mathcal{V}_{\nu}^{(\nu)}-\ldots \mu \leftrightarrow \nu\right]-\epsilon_{\mu \nu \lambda \nu^{\prime}}\left(K-2 \mathcal{J}^{(e)}\right)^{\lambda} \mathcal{V}^{(\nu) \nu^{\prime}} & =0,
\end{aligned}
$$


using $c_{V}^{(\nu)}+c_{A}^{(\nu)}=2$; here $K_{\mu} \equiv p_{\mu}+\frac{i \hbar}{2} \partial_{x^{\mu}}$. Separating real and imaginary parts, we expand the resulting equations in powers of $\hbar$ :

$$
\begin{aligned}
\left(p-2 J^{(e)}\right) \cdot \mathcal{V}^{(\nu)}+O\left(\hbar^{2}\right) & =0, \\
\left(\partial_{x}+2 \partial_{x} \cdot \partial_{p} J^{(e)}\right) \cdot \mathcal{V}^{(\nu)}+O\left(\hbar^{2}\right) & =0, \\
{\left[\left(p-2 J^{(e)}\right)_{\mu} \mathcal{V}_{\nu}^{(\nu)}-\ldots \mu \leftrightarrow \nu\right]+O(\hbar) } & =0, \\
\epsilon_{\mu \nu \lambda \nu^{\prime}}\left(p-2 J^{(e)}\right)^{\lambda} \mathcal{V}^{(\nu) \nu^{\prime}}+O(\hbar) & =0,
\end{aligned}
$$

thus proceeding similarly as in the case of the electron-positron plasma up to this point.

However, now it is obvious that the following Ansatz immediately solves Eqs. (53) and (54):

$$
\mathcal{V}_{\mu}^{(\nu)} \equiv\left(p-2 J^{(e)}\right)_{\mu} \tilde{f}^{(\nu)}
$$

with a scalar function $\tilde{f}$ of the phase space variables $x, p$. Furthermore, to leading order in $\hbar$, it converts Eq. (49) into the mass-shell constraint:

$$
\left(p-2 J^{(e)}\right)^{2} \tilde{f}^{(\nu)}=0
$$

which demonstrates that it is the kinetic momentum, $k_{\mu} \equiv p_{\mu}-2 J^{(e)}(x)$, which is to be on-shell here.

Performing analogous steps as in Eqs. (42)-(44) before, redefining $\tilde{f}^{(\nu)}(x, p) \equiv f^{(\nu)}(x, k)$ in particular, we obtain directly the $\nu_{L} \bar{\nu}_{R}$ mass-shell constraint:

$$
k^{2} f^{(\nu)}=0
$$

and from Eq. (52) the Vlasov type transport equation for the $\nu_{L} \bar{\nu}_{R}$ density function:

$$
\left(k \cdot \partial_{x}-2 k_{\mu}\left[\partial_{x}^{\mu} J^{(e) \nu}-\partial_{x}^{\nu} J^{(e) \mu}\right] \partial_{k^{\nu}}\right) f^{(\nu)}=0,
$$

which may be compared to Eqs. (45) and (46) of the electron-positron plasma.

In order to complete the set of coupled classical transport and constraint equations (45), (46), (57), and (58), we have to reconsider the four-currents entering here and into the Maxwell equation (23) in the limit $\hbar \rightarrow 0$.

Implementing the spin saturation, in particular $\mathcal{A}_{\mu}^{(e)} \equiv 0$, and using Eqs. (37) and (43), we obtain the weak $e^{+} e^{-}$current:

$$
J_{\mu}^{(e)}(x)=4 \frac{G_{F}}{\sqrt{2}} \frac{c_{V}}{m^{(e)}} \int \mathrm{d}^{4} p\left(p_{\mu}-c_{V} J_{\mu}^{(\nu)}(x)\right) \tilde{f}^{(e)}(x, p)=4 \frac{c_{V} G_{F}}{\sqrt{2}} \int \mathrm{d}^{4} k \frac{k_{\mu}}{m^{(e)}} f^{(e)}(x, k),
$$

cf. Eq. (13), with $c_{V}^{(e)}=c_{V}$. Similarly, the electromagnetic current assumes the form:

$$
J_{\mathrm{em}}^{\mu}(x)=4 e \int \mathrm{d}^{4} k \frac{k_{\mu}}{m_{e}} f^{e}(x, k)
$$

cf. Eq. (23). Finally, the weak $\nu_{L} \bar{\nu}_{R}$ current is:

$$
J_{\mu}^{(\nu)}(x)=8 \frac{G_{F}}{\sqrt{2}} \int \mathrm{d}^{4} p\left(p_{\mu}-2 J_{\mu}^{(e)}(x)\right) \tilde{f}^{(\nu)}(x, p)=8 \frac{G_{F}}{\sqrt{2}} \int \mathrm{d}^{4} k k_{\mu} f^{(\nu)}(x, k),
$$

using once more $\mathcal{V}_{\mu}^{(\nu)}=\mathcal{A}_{\mu}^{(\nu)}, c_{V}^{(\nu)}+c_{A}^{(\nu)}=2$, as well as Eq. (55). 
The closed set of four coupled mass-shell constraint and transport equations, with the currents determined by scalar (density) functions, along with the Maxwell equation present the final result of our derivation of the semiclassical nonequilibrium transport theory of neutrinos and electrons. It incorporates their antiparticles as discussed in more detail for the QED case in Ref. [11], as well as electromagnetic fields, assuming an $e^{+} e^{-}$-spin saturated system in the mean field dominated regime.

We note that the structure of our final closed set of equations could essentially be anticipated from purely classical kinetic theory considerations, as previously observed [6, 12]. On the other hand, for the study of spin-polarization or strong magnetic field effects and higher order quantum corrections, we must go back to our previous set of Eqs. (15)-(19) of Section 2.1 .

\section{Linear Response Analysis and (Un)Stable Collective Modes}

Presently, we apply the transport theory of Section 2, in order to derive the semiclassical dispersion relations of collective modes of a neutrino-electron system in general (Section 3.1).

In Section 3.2 we specialize our results for the Type II supernova scenario. The relevant distribution functions are introduced in Section 3.2.1 and the necessary response functions calculated in Section 3.2 .2 .

In Section 3.2.3 we evaluate the dispersion relations for various collective modes. We determine their (in)stability properties in the neutrino 'beam' plus electron 'plasma sphere' system formed during a Type II supernova explosion. The final Section 3.2.4 is devoted to a discussion of the validity of the approximations used.

\subsection{The Linear Response Theory for Neutrino-Electron Systems}

The behavior of collective modes, in particular, the onset of instabilities, is determined by the evolution of small perturbations of a generic set of stationary distribution functions, which may be caused by scattering interactions, for example. Therefore, we write the scalar density distributions in the form:

$$
f^{(l)}(x, k)=f_{S}^{(l)}(k)+\delta f^{(l)}(x, k)
$$

where $f_{S}^{(l)}$ denotes the assumed homogeneous four-momentum dependent solutions of the mass-shell and transport equations and $\delta f^{(l)}$ an initially small perturbation. This assumption of homogeneity greatly simplifies the subsequent analysis and describes a sufficiently large 'free-streaming' electronneutrino system.

The weak currents $J_{\mu}^{(l)}$ determined by $f_{S}^{(l)}$, cf. Eqs. (59) and (61), are homogeneous and the antisymmetric tensors which enter the transport equations (46) and (58),

$$
G^{(l) \mu \nu} \equiv c^{(l)}\left[\partial_{x}^{\mu} J^{\left(l^{\prime}\right) \nu}-\partial_{x}^{\nu} J^{\left(l^{\prime}\right) \mu}\right],
$$

vanish in this case; from here on $c^{(e)} \equiv c_{V}$ and $c^{(\nu)} \equiv 2$. Furthermore, assuming an isotropic on-shell electron-positron distribution,

$$
f_{S}^{(e)}(k) \equiv \delta\left[k^{2}-m^{(e) 2}-c_{A}^{2} J^{(\nu)} \cdot J^{(\nu)}\right] f^{(e)}\left(k^{0},|\vec{k}|\right),
$$

cf. Eq. (45), it follows that the corresponding electromagnetic four-current (60) vanishes, if we additionally assume a neutralizing background charge or approximately equal densities of electrons and positrons, depending on the circumstances. Consistently we set $F^{\mu \nu} \equiv 0$, i.e., considering a situation without external electromagnetic fields.

Indeed, then, the initial on-shell distributions $f_{S}^{(\nu)}(k)$ and $f_{S}^{(e)}(k)$ are stationary in the absence of collisions. They will be further specified shortly. 
Linearizing the transport equations (46) and (58) with respect to the small perturbations $\delta f^{(l)}$, we obtain for the electrons:

$$
i k \cdot q \delta f^{(e)}(q, k)+k_{\mu}\left(\delta G^{(e) \mu \nu}(q)+e \delta F^{\mu \nu}(q)\right) \partial_{k^{\nu}} f_{S}^{(e)}(k)=0
$$

where we introduced the Fourier transform for any function $g$ of the space-time coordinates, $g(x) \equiv$ $(2 \pi)^{-4} \int \mathrm{d}^{4} q \exp (-i q \cdot x) g(q)$. Here $\delta G^{(e)}$ and $\delta F$ denote the weak and electromagnetic tensors induced by the perturbations $\delta f^{(\nu)}$ and $\delta f^{(e)}$, respectively. From Eq. 63) we obtain:

$$
\delta G^{(l) \mu \nu}(q)=-i c^{(l)}\left(q^{\mu} g^{\nu \lambda}-q^{\nu} g^{\mu \lambda}\right) \delta J_{\lambda}^{\left(l^{\prime}\right)} .
$$

Furthermore, solving the Maxwell equation (23) with the retarded boundary condition (damping in the infinite past), we obtain:

$$
e \delta F^{\mu \nu}(q)=\frac{i e}{q^{2}+i \epsilon q^{0}}\left(q^{\mu} g_{\lambda}^{\nu}-q^{\nu} g_{\lambda}^{\mu}\right) \delta J_{\mathrm{em}}^{\lambda}=i \frac{\sqrt{2} e^{2}}{c_{V} G_{F}} \frac{1}{q^{2}+i \epsilon q^{0}}\left(q^{\mu} g^{\nu \lambda}-q^{\nu} g^{\mu \lambda}\right) \delta J_{\lambda}^{(e)},
$$

where $\epsilon \rightarrow 0^{+}$, and where we used Eqs. (59) and (60), in order to express the (conserved) electromagnetic current fluctuation in terms of its weak counterpart.

Implementing the retarded boundary condition, i.e. the 'Landau prescription' [9], the electron transport equation (65) is solved by:

$$
\delta f^{(e)}(q, k)=\frac{k_{\mu}\left(\delta G^{(e) \mu \nu}(q)+e \delta F^{\mu \nu}(q)\right) \partial_{k^{\nu}}}{-i\left(k \cdot q+i \epsilon k^{0}\right)} f_{S}^{(e)}(k)
$$

Similarly, the perturbation of the stationary neutrino distribution is determined by:

$$
\delta f^{(\nu)}(q, k)=\frac{k_{\mu} \delta G^{(\nu) \mu \nu}(q) \partial_{k^{\nu}}}{-i\left(k \cdot q+i \epsilon k^{0}\right)} f_{S}^{(\nu)}(k)
$$

Obviously, Eqs. (68) and (69) are coupled to each other via Eqs. (66).

We proceed by introducing the response functions:

$$
M^{(l) \lambda \rho}(q) \equiv 4 \int \mathrm{d}^{4} k \frac{k^{\lambda}}{m^{(l=e)}} \frac{1}{k \cdot q+i \epsilon k^{0}}\left(k \cdot q \partial_{k}^{\rho}-k^{\rho} q \cdot \partial_{k}\right) f_{S}^{(l)}(k),
$$

which will be calculated for specific choices of the stationary distributions $f_{S}^{(l)}$ shortly; the factor $1 / m^{(l=e)}$ is meant to apply only in the $e^{+} e^{-}$case and to be replaced by 1 for the (approximately) massless $\nu_{L} \bar{\nu}_{R}$ case.

Making use of the response functions, we multiply Eqs. (68) and (69) by the appropriate factors, cf. Eqs. (59) and (61), and integrate over $\mathrm{d}^{4} k$, in order to obtain a closed set of algebraic equations:

$$
\begin{aligned}
\delta J^{(e) \lambda}(q) & =M^{(e) \lambda \rho}(q)\left(\frac{c_{V}^{2} G_{F}}{\sqrt{2}} \delta J_{\rho}^{(\nu)}(q)-\frac{e^{2}}{q^{2}+i \epsilon q^{0}} \delta J_{\rho}^{(e)}(q)\right) \\
\delta J^{(\nu) \lambda}(q) & =\frac{4 G_{F}}{\sqrt{2}} M^{(\nu) \lambda \rho}(q) \delta J_{\rho}^{(e)}(q)
\end{aligned}
$$

where the tensor fluctuations $\delta G^{(l)}$ and $\delta F$ were eliminated with the help of Eqs. (66) and (67), respectively. Inserting the second into the first equation, the final result is:

$$
\mathcal{M}^{(e) \lambda \rho}(q) \delta J_{\rho}^{(e)}(q) \equiv\left[g^{\lambda \rho}+M^{(e) \lambda \sigma}(q)\left(\frac{e^{2}}{q^{2}+i \epsilon q^{0}} g_{\sigma}^{\rho}-2 c_{V}^{2} G_{F}^{2} g_{\sigma \tau} M^{(\nu) \tau \rho}(q)\right)\right] \delta J_{\rho}^{(e)}(q)=0
$$


The solvability condition of this vector equation determines the dispersion relation for the perturbations of the stationary electron-positron distribution:

$$
\operatorname{Det} \mathcal{M}^{(e)}(q)=0
$$

where $\mathcal{M}^{(e)}$ is a $4 \times 4$-matrix in the Lorentz indices. Analogously one obtains the dispersion relation for the neutrino case, which we do not pursue.

A final remark is in order here. From the structure of Eq. (73), particularly the generically small weak coupling term compared with the electromagnetic one, it is natural to expect that the neutrinos can only influence the resulting dispersion relations noticeably, if their response function shows rather singular behavior. Furthermore, in this case, weak and electromagnetic interactions presumably will mix in the corresponding collective modes, due to the products involved, for example, in Eqs. (73) and (74). This will be studied in the following sections with the application to a supernova scenario.

\subsection{The Supernova Two-Stream Scenario}

The above results are fairly general and need to be specialized according to the physical nature of the stationary distributions as well as of their potentially unstable perturbations. We shall now study the idealized situation where an electrically neutral finite temperature electron-positron plasma is hit by a neutrino-antineutrino beam. (Anti-)neutrinos are radiated from the neutrino sphere, move approximately radially outwards, and interact collectively with the electron-positron plasma sphere forming the 'radiation bubble' in Bethe's supernova scenario [2]. As before, we derive our results in the collisionless limit.

Typically, it is assumed that a short-lived, but intense neutrino flux $\left(3 \times 10^{29} \mathrm{~W} / \mathrm{cm}^{2}\right.$, total integrated luminosity up to the order of several $10^{53} \mathrm{erg}$ ) with an approximately thermal spectrum corresponding to a temperature $T_{\nu} \approx 1 \ldots 10 \mathrm{MeV}$ is released from the collapsing core and interacts at a distance of about $30 \ldots 300 \mathrm{~km}$ from the center with the surrounding moderately relativistic electron plasma of (charge) density $n_{e} \lesssim 10^{30} \mathrm{~cm}^{-3}$ and temperature $T_{e} \gtrsim 0.5 \mathrm{MeV}$; here the uncertainties mostly reflect differing scenarios considered in this context [1, 2, 3, 5, 6]. We will study a corresponding set of parameters, following the discussion of the radiation bubble by Bethe [2].

For the above (optimistic charge) density and temperature, the electron-positron plasma is nondegenerate, with an estimated chemical potential $\mu_{e}<\pi T$. This is indirectly supported by Bethe's results, see in particular Sections VI. E-G of Ref. [2], which demonstrate the dilute character of matter in the radiation bubble - the energy or entropy density of the 'radiation' (i.e. of photons plus pairwise produced electrons and positrons) is more than a factor $10^{2}$ higher than that of nucleons in the bubble. Therefore, any background charge contamination by protons must be small here, and correspondingly the net charge of electrons over positrons neutralizing the plasma. Hence we may neglect the finite electron chemical potential in a first approximation.

\subsubsection{Distribution Functions}

The following stationary electron-positron distribution will now be considered, cf. Eq. 64):

$$
f_{S}^{(e)}(k)=(2 \pi)^{-3} m^{(e)} \delta\left[k^{2}-m^{(e) 2}-c_{A}^{2} J^{(\nu)} \cdot J^{(\nu)}\right]\left(\Theta\left(k^{0}\right) F\left(k^{0} / T_{e}\right)+\Theta\left(-k^{0}\right) F\left(-k^{0} / T_{e}\right)\right),
$$

where $F(x) \equiv\left(\mathrm{e}^{x}+1\right)^{-1}$, and where $T_{e}$ denotes their temperature. When $J^{(\nu)}=0$, Eq. (75) describes the $e^{+} e^{-}$blackbody radiation (omitting the vacuum contribution). We remark that antiparticles are represented as fermions with negative four-momentum here [11].

Concerning the emission from the neutrino sphere, we neglect its collective flow relative to the electron-positron plasma sphere, or vice versa. However, it is important to incorporate the dilution 
and angular squeezing effects due to the spherical geometry. Thus, we assume the following stationary (approximately massless) neutrino-antineutrino distribution:

$$
f_{S}^{(\nu)}(k)=\frac{1}{2}(2 \pi)^{-3} \delta\left[k^{2}\right]\left(\Theta\left(k^{0}\right) \Theta\left(\theta_{\max }-\theta_{\vec{n}, \vec{k}}\right) F\left(\left[k^{0}-\mu_{\nu}\right] / T_{\nu}\right)+\ldots k, \mu_{\nu} \rightarrow-k,-\mu_{\nu}\right),
$$

where $\mu_{\nu}$ denotes their chemical potential and only one $\left(\nu_{L}\right.$ or $\left.\bar{\nu}_{R}\right)$ spin state is taken into account. The additional $\Theta$-function, implementing the radial ('outward') unit vector $\vec{n}$, accounts for the finite opening angle $\theta_{\vec{n}, \vec{k}}$ between neutrino momenta and the radial direction. The maximal opening angle is determined by $\sin \theta_{\max }=R / r$, where $r$ denotes the distance from the center of the neutrino sphere of radius $R$. The usual dilution factor, $d \equiv(R / r)^{2}=\sin ^{2} \theta_{\text {max }}$, does not appear explicitly, but is recovered in the calculation of, for example, the energy flux from the neutrino sphere based on $f_{S}^{(\nu)}$.

We remark that the neutrino distribution is not necessarily uniform within the cone defined by $\theta_{\max }$. It may vary considerably, depending on the emission characteristics of the neutrino sphere. Thus, the distribution of Eq. (76) may represent an opening angle average; the (ir)relevance of the sharp $\Theta$-function cut-off will be discussed in the final subsection 3.2.4 . Furthermore, it is not a global solution of the spherical free-streaming problem. However, our approximate spatially homogeneous distribution, with the parametric dependence on $R / r$, is sufficient for the study of collective modes with a characteristic wavelength very much less than $R$, even though we are interested in the longwavelength limit with respect to the microscopic scales.

We omit the $\mu$ - and $\tau$-neutrinos at present which have a considerably weaker effective coupling, see Eq. (73) together with the remarks after Eq. (1); using $\sin ^{2} \theta_{W} \approx 0.23$, we have $c_{V} \approx 0.96(-0.04)$ for the electron $(\mu, \tau-)$ neutrinos. Furthermore, their chemical potential vanishes. For the electron (anti-)neutrinos, which carry about $4 / 10$ of the total neutrino energy flux, we adopt Bethe's estimate which yields $\eta_{\nu} \equiv \mu_{\nu} / T_{\nu}=0.29$ [2]

Next, we proceed to calculate the energy-momentum tensor, similarly as in Ref. [11], for a stationary free neutrino-antineutrino distribution:

$$
T_{\mu \nu}^{(\nu)}(x)=\operatorname{tr} \int \mathrm{d}^{4} k k_{\nu} \gamma_{\mu} W^{(\nu)}(x, k)=4 \int \mathrm{d}^{4} k k_{\nu} \mathcal{V}_{\mu}^{(\nu)}(x, k),
$$

i.e. in terms of the vector density, cf. Eq. (10). Employing Eq. (55) and projecting on the 'outward' momentum direction, we obtain the electron- $\nu_{L} \bar{\nu}_{R}$ energy flux corresponding to the homogeneous equilibrium distribution (76):

$$
\begin{aligned}
T^{(\nu) 0 i} n^{i} & =4 \int \mathrm{d}^{4} k k^{0} k^{z} f_{S}^{(\nu)}(k)=\frac{d}{8 \pi^{2}} \int_{0}^{\infty} \mathrm{d} k k^{3}\left(F\left(\left[k-\mu_{\nu}\right] / T_{\nu}\right)+F\left(\left[k+\mu_{\nu}\right] / T_{\nu}\right)\right) \\
& =\frac{7 \pi^{2}}{480} d T_{\nu}^{4}\left(1+\frac{30}{7 \pi^{2}} \eta_{\nu}^{2}+\frac{15}{7 \pi^{4}} \eta_{\nu}^{4}\right)=\frac{4}{10} \cdot \frac{d L}{\pi R^{2}},
\end{aligned}
$$

choosing $\vec{n}=(0,0,1)$, and where $L$ denotes the total neutrino-plus-antineutrino luminosity. The integral is evaluated exactly with the help of a formula from Ref. [18]. Correction terms involving powers of $m^{(\nu)} /\left(\eta_{\nu} T_{\nu}\right)$ would be completely negligible for temperatures in the $\mathrm{MeV}$ range and a typical neutrino mass (much) less than $1 \mathrm{eV}$. The last equality in Eq. (78) provides the relation between temperature and radius of the neutrino sphere, given its luminosity $L$ [2].

Similarly, we obtain from Eq. (61) by direct calculation:

$$
J^{(\nu) \mu}=8 \frac{G_{F}}{\sqrt{2}} \int \mathrm{d}^{4} k k^{\mu} f_{S}^{(\nu)}(k)=\frac{G_{F}}{\sqrt{2}} \frac{d T_{\nu}^{3}}{12}\left(\eta_{\nu}+\frac{1}{\pi^{2}} \eta_{\nu}^{3}\right) \xi^{\mu},
$$

where $\xi^{\mu} \equiv\left(2 /\left[1+\cos \theta_{\max }\right], 0,0,1\right)$. As expected, the neutrino current components are very small, since for temperatures of about $10 \mathrm{MeV}$ we have $G_{F} T_{\nu}{ }^{3} \approx 10^{-8} \mathrm{MeV}$. Therefore, the corresponding term $\propto J^{(\nu)} \cdot J^{(\nu)}$ in the expression for the stationary electron-positron distribution, cf. Eq. (75), can be safely neglected henceforth. 


\subsubsection{Response Functions}

After specifying the unperturbed stationary electron and neutrino distributions, $f_{S}^{(e)}$ and $f_{S}^{(\nu)}$ respectively, we calculate the response functions $M^{(e) \lambda \rho}$ and $M^{(\nu) \lambda \rho}$ defined in Eq. (70). For the following calculations it is convenient to perform a partial integration, which yields:

$$
M^{(l) \lambda \rho}(q)=4 \int \mathrm{d}^{4} k \frac{f_{S}^{(l)}(k)}{m^{(l=e)}}\left(-g^{\lambda \rho}+\frac{q^{\lambda} k^{\rho}+q^{\rho} k^{\lambda}}{k \cdot q+i \epsilon k^{0}}-\frac{q^{2} k^{\lambda} k^{\rho}}{\left(k \cdot q+i \epsilon k^{0}\right)^{2}}\right),
$$

which is now obviously symmetric and transverse, $q_{\lambda} M^{(l) \lambda \rho}(q)=0$. Thus, the current fluctuations are properly conserved, $q_{\lambda} \delta J^{l \lambda}(q)=0$, cf. Eqs. (71)-(72).

Beginning with the electron case, the calculation is facilitated by recalling that the distribution function $f_{S}^{(e)}$, Eq. (75), is isotropic with respect to the three-momentum components. Therefore, the spatial part of the response function can be decomposed into a transverse and a longitudinal part,

$$
M^{(e) i j}(q) \equiv\left(\delta^{i j}-\frac{q^{i} q^{j}}{\vec{q}^{2}}\right) M_{T}^{(e)}(q)+\frac{q^{i} q^{j}}{\vec{q}^{2}} M_{L}^{(e)}(q) .
$$

Defining the electric (Debye) screening mass,

$$
m_{D}^{2} \equiv \frac{4 e^{2}}{\pi^{2}} \int_{0}^{\infty} \mathrm{d} k k F\left(k / T_{e}\right)=\frac{1}{3} e^{2} T_{e}^{2},
$$

the results of a standard calculation for the electron-positron response function are:

$$
\begin{aligned}
-e^{2} M^{(e) 00}\left(q^{0}, \vec{q}\right) & =m_{D}^{2}\left[1-\frac{1}{2} \frac{\omega}{q}\left(\ln \left|\frac{q+\omega}{q-\omega}\right|-i \pi \Theta(q-\omega)\right)\right], \\
M^{(e) 0 i}\left(q^{0}, \vec{q}\right) & =M^{(e) i 0}\left(q^{0}, \vec{q}\right)=\frac{q^{0} q^{i}}{q^{2}} M^{(e) 00}\left(q^{0}, \vec{q}\right), \\
M_{L}^{(e)}\left(q^{0}, \vec{q}\right) & =\frac{\omega^{2}}{q^{2}} M^{(e) 00}\left(q^{0}, \vec{q}\right), \\
e^{2} M_{T}^{(e)}\left(q^{0}, \vec{q}\right) & =\frac{1}{2} m_{D}^{2} \frac{\omega^{2}}{q^{2}}\left[1-\frac{1}{2}\left(\frac{\omega}{q}-\frac{q}{\omega}\right)\left(\ln \left|\frac{q+\omega}{q-\omega}\right|-i \pi \Theta(q-\omega)\right)\right],
\end{aligned}
$$

where we implemented the relativistic limit and neglected correction terms in powers of $m_{e} / T_{e}$; here we simplified the notation by introducing $\omega \equiv\left|q^{0}\right|$ and $q \equiv|\vec{q}|$. We note the appearance of the imaginary parts which, in general, are responsible for Landau damping [9, 19]. These kinetic theory results completely agree with the perturbative one-loop evaluation of the QED polarization tensor in the high-temperature limit [20], a correspondence which was has been observed in many other cases, e.g., [12].

In a fully realistic calculation, corrections due to the finite ratio of $m_{e} / T_{e} \lesssim 1$ should also be considered. We neglect them in our present work, since the appropriate electron temperature is not precisely known in this context. Furthermore, unfortunately, this would necessitate numerical calculations where the transparency of the analytical results presented here would be lost. On the one hand, it seems unlikely that the additional mass scale can qualitatively change any of our conclusions, since it is well separated from all the plasma scales entering in the following. However, for particular effects, e.g. proper Landau damping [9, 19], a finite mass may be crucial (cf. footnote following the discussion after Eq. (112)). Thus, proper 'neutrino Landau damping' has been discussed in more detail by Silva et al. recently [5].

Next, we turn to the calculation of the neutrino response function $M^{(\nu) \lambda \rho}$. It is more involved due to the preferred direction of propagation, which enters here through the dependence of the stationary 
distribution (76) on the 'radial' unit vector $\vec{n}$. In order to facilitate our task, we consider two cases separately, depending on the orientation of the wave vector $\vec{q}$ with respect to $\vec{n}$ : $\vec{q} \| \vec{n}$ (Case I) and $\vec{q} \perp \vec{n}$ (Case II). We recall that $\vec{q}$ determines the direction of propagation of the collective excitations of the electron-positron plasma, especially in the presence of the neutrino flux.

Case I. Here we expect a response function with a formal structure generalizing the familiar results of Eqs. (83)-(86), since the geometry determining the essential angular integrations is identical to the previous case. Therefore, a tensor decomposition into transverse and longitudinal parts analogous to Eq. (81) still applies. However, the maximal opening angle $\theta_{\max }$ between neutrino momenta and the 'radial' direction limits the azimuthal angle $\theta$, e.g. in the vector decomposition,

$$
\vec{k}=\overrightarrow{q q}^{-1} k \cos \theta+\vec{k}_{\perp}
$$

with $k \equiv|\vec{k}|$, which is conveniently employed after converting the integral of Eq. (80) to the corresponding threedimensional (on-shell) form.

Furthermore, instead of the Debye mass of Eq. (82), we introduce the weak thermal mass:

$$
m_{w}^{2} \equiv \frac{2 c_{V}^{2}}{\pi^{2}} \int_{0}^{\infty} \mathrm{d} k k\left(F\left(\left[k-\mu_{\nu}\right] / T_{\nu}\right)+F\left(\left[k+\mu_{\nu}\right] / T_{\nu}\right)\right)=\frac{1}{3} c_{V}^{2} T_{\nu}^{2}\left(1+\frac{3}{\pi^{2}} \eta_{\nu}^{2}\right)
$$

which takes the finite chemical potential of the neutrinos into account. Here we applied the ultrarelativistic limit discussed before, as well as the appropriate integral formula from Ref. [18].

Then, we obtain the components of the neutrino-antineutrino response function $(\vec{q} \| \vec{n})$ :

$$
\begin{aligned}
&-2 c_{V}^{2} M^{(\nu) 00}\left(q^{0}, \vec{q}\right)= m_{w}^{2}\left[\frac{1-z}{4}\left(1-\frac{q+q^{0}}{z q-q^{0}}\right)\right. \\
&\left.-\frac{1}{2} \frac{q^{0}}{q}\left(\ln \left|\frac{z q-q^{0}}{q-q^{0}}\right|-i \pi \Theta\left(q-q^{0}\right)+i \pi \Theta\left(z q-q^{0}\right)\right)\right], \\
& M^{(\nu) 0 i}\left(q^{0}, \vec{q}\right)= M^{(\nu) i 0}\left(q^{0}, \vec{q}\right)=\frac{q^{0} q^{i}}{q^{2}} M^{(\nu) 00}\left(q^{0}, \vec{q}\right), \\
& M_{L}^{(\nu)}\left(q^{0}, \vec{q}\right)= \frac{\omega^{2}}{q^{2}} M^{(\nu) 00}\left(q^{0}, \vec{q}\right), \\
& 2 c_{V}^{2} M_{T}^{(\nu)}\left(q^{0}, \vec{q}\right)= \frac{1}{2} m_{w}^{2} \frac{\omega^{2}}{q^{2}}\left[\frac{1-z}{2}\left(1-\frac{1+z}{2} \frac{q\left(1-q^{2} / \omega^{2}\right)}{z q-q^{0}}\right)\right. \\
&\left.-\frac{1}{2}\left(\frac{q^{0}}{q}-\frac{q}{q^{0}}\right)\left(\ln \left|\frac{z q-q^{0}}{q-q^{0}}\right|-i \pi \Theta\left(q-q^{0}\right)+i \pi \Theta\left(z q-q^{0}\right)\right)\right]
\end{aligned}
$$

where $z \equiv \cos \theta_{\max }$ and $\omega^{2} \equiv\left(q^{0}\right)^{2}$. Indeed, for $z \rightarrow-1$, i.e. without restriction on the opening angle, we recover the formal structure of Eqs. (83)-(86), while for $z \rightarrow 1$ the response function vanishes.

Furthermore, we observe that for a finite opening angle $(1>z>-1)$ the Landau damping imaginary parts are limited to the region $q>q^{0}>z q$ and that at the resonance frequency $q^{0}=\Omega_{\|} \equiv z q$ the response function has additional singularities, which are absent for $z=-1$. In the present case, with $\vec{q} \| \vec{n}$, the longitudinal as well as the transverse components, $M_{L}^{(\nu)}$ and $M_{T}^{(\nu)}$ respectively, are affected.

Case II. In this case, with $\vec{q} \perp \vec{n}$, we introduce a third unit vector $\vec{e}$, perpendicular to the other two vectors, in order to decompose the momentum vector for the threedimensional response function integral,

$$
\vec{k}=k\left(\vec{n} \cos \theta+\overrightarrow{q q^{-1}} \sin \theta \cos \phi+\vec{e} \sin \theta \sin \phi\right),
$$


with the azimuthal and polar angles $\theta$ and $\phi$, respectively, such that $\vec{k} \cdot \vec{q}=k q \sin \theta \cos \phi$. The resulting angular integrations can all be done analytically in the appropriate ultrarelativistic limit, either by elementary or contour integration techniques.

Due to the symmetry properties of the required integrals, presently it turns out to be useful to decompose the spatial part of the response function as follows:

$$
M^{(\nu) i j}(q) \equiv\left(\delta^{i j}-n^{i} n^{j}-\frac{q^{i} q^{j}}{\vec{q}^{2}}\right) M_{T}^{(\nu)}(q)+n^{i} n^{j} M_{L_{1}}^{(\nu)}(q)+\frac{q^{i} q^{j}}{\vec{q}^{2}} M_{L_{2}}^{(\nu)}(q)+\frac{n^{i} q^{j}+n^{j} q^{i}}{|\vec{q}|} M_{3}^{(\nu)}(q) .
$$

All other terms which could arise vanish identically, since the corresponding polar angle integration comprises an odd function.

In order to check the ensuing lengthy calculations, we also evaluated independently the integrals resulting from the transversality condition mentioned after Eq. 80$), q_{\lambda} M^{(\nu) \lambda \rho}(q)=0$, as well as from the trace $M_{\lambda}^{(\nu) \lambda}(q)$. These results we compared with what is obtained using the calculated components of the response function in the following. In fact, this procedure leads to considerable simplifications.

Then, for $0 \leq \theta_{\max } \leq \pi / 2$, i.e. $0 \leq z \leq 1$, we finally obtain these components of the neutrinoantineutrino response function $(\vec{q} \perp \vec{n})$ :

$$
\begin{aligned}
& -2 c_{V}^{2} M^{(\nu) 00}\left(q^{0}, \vec{q}\right)=m_{w}^{2}\left[\frac{1 \pm 1-z}{4} \mp \frac{z}{4} \frac{q^{0}}{\sqrt{q_{z}^{2}}} \mp \frac{1}{2} \frac{q^{0}}{q} \ln \left(\frac{q+q^{0}}{z q+\sqrt{q_{z}^{2}}}\right)\right], \\
& M^{(\nu) 0 i}\left(q^{0}, \vec{q}\right)=M^{(\nu) i 0}\left(q^{0}, \vec{q}\right)=\frac{q n^{i}}{q^{0}} M_{3}^{(\nu)}\left(q^{0}, \vec{q}\right)+\frac{q^{0} q^{i}}{q^{2}} M^{(\nu) 00}\left(q^{0}, \vec{q}\right), \\
& 2 c_{V}^{2} M_{T}^{(\nu)}\left(q^{0}, \vec{q}\right)=\frac{1}{2} m_{w}^{2} \frac{\left(q^{0}\right)^{2}}{q^{2}}\left[\frac{1-z}{2} \mp \frac{1}{2}\left(\frac{q^{0}}{q}-\frac{q}{q^{0}}\right) \ln \left(\frac{q+q^{0}}{z q+\sqrt{q_{z}^{2}}}\right)\right], \\
& 2 c_{V}^{2} M_{L 1}^{(\nu)}\left(q^{0}, \vec{q}\right)=\frac{1}{4} m_{w}^{2}\left[1-z \pm\left(\frac{\left(q^{0}\right)^{2}}{q^{2}}-1\right)\left(1-z \frac{q^{0}}{\sqrt{q_{z}^{2}}}-\frac{q^{0}}{q} \ln \left(\frac{q+q^{0}}{z q+\sqrt{q_{z}^{2}}}\right)\right)\right] \text {, } \\
& M_{L 2}^{(\nu)}\left(q^{0}, \vec{q}\right)=\frac{\left(q^{0}\right)^{2}}{q^{2}} M^{(\nu) 00}\left(q^{0}, \vec{q}\right) \\
& 2 c_{V}^{2} M_{3}^{(\nu)}\left(q^{0}, \vec{q}\right)= \pm \frac{1}{4} m_{w}^{2} \frac{q^{0}}{q}\left[\frac{\left(q^{0}\right)^{2}}{q^{2}}\left(1-\left(q^{0}\right)^{-1} \sqrt{q_{z}^{2}}\right)+\left(\frac{\left(q^{0}\right)^{2}}{q^{2}}-1\right)\left(1-q^{0} / \sqrt{q_{z}^{2}}\right)\right]
\end{aligned}
$$

where we introduced the abbreviation,

$$
q_{z}^{2} \equiv\left(q^{0}\right)^{2}-q^{2}\left(1-z^{2}\right)
$$

with $q \equiv|\vec{q}|$ and $z \equiv \cos \theta_{\max }$, as before. Several qualifying remarks are in order here:

- For later convenience we did not separate real and imaginary parts in Eqs. (95)-(100) which are valid for complex $q^{0} \equiv \omega+i \gamma$, provided the imaginary part here is sufficiently small, $|\gamma| \ll|\omega|$, or infinitesimal.

- Either the upper or the lower signs have to chosen consistently in Eqs. (95)-(100) according to the following rules $\left(0 \leq \theta_{\max } \leq \pi / 2\right)$ :

$$
\begin{aligned}
& \gamma>0 \text { and } \omega>0 \Rightarrow \text { upper signs } ; \quad \gamma>0 \text { and } \omega<-q \sin \theta_{\max } \Rightarrow \text { lower signs ; } \\
& \gamma<0 \text { and } \omega<0 \Rightarrow \text { lower signs } ; \quad \gamma<0 \text { and } \omega>q \sin \theta_{\max } \Rightarrow \text { upper signs }
\end{aligned}
$$

They are due to the (angular) contour integrations, which result in different contributions according to the listed rules. 
We do not report the results for $\omega$ in the intervals which are excluded in (102), since the azimuthal angle integrations have to be split in this case, yielding even more complicated expressions.

Obviously, the response function has additional square-root singularities at the resonance frequencies $\omega=\Omega_{\perp}^{ \pm} \equiv \pm q \sin \theta_{\text {max }}$, as $\gamma \rightarrow 0$. The transverse component $M_{T}^{(\nu)}$, however, is not affected in the present Case II $(\vec{q} \perp \vec{n})$.

This completes the calculation of the response functions for the model distributions discussed in the previous subsection.

\subsubsection{Dispersion Relations, Collective Modes and Instabilities}

It is useful to begin the study of the dispersion relations following from Eqs. (73)-(74) with the case of the electromagnetically interacting electron-positron plasma, i.e. with the weak interaction term $\propto G_{F}^{2}$ in Eq. (73) switched off.

Considering separately transverse (' $T$ ') and longitudinal (' $L$ ') current fluctuations, i.e. $\delta \vec{J}(e)(q) \perp \vec{q}$ and $\delta \vec{J}^{(e)}(q) \| \vec{q}$, respectively, Eq. (74) yields two equations determining the corresponding dispersion relations:

$$
\begin{array}{ll}
T: & \left(1-e^{2} M_{T}^{(e)} /\left(q^{2}+i \epsilon q^{0}\right)\right)^{3}=0, \\
L: & 1-e^{2} M^{(e) 00} / \vec{q}^{2}=0,
\end{array}
$$

with the plasma response functions of Eqs. (83)-(86), and where the decomposition (81) of the spatial part of the response function is especially taken into account.

The real solutions with $\omega \equiv\left|q^{0}\right|>q \equiv|\vec{q}|$ of Eqs. (103) and (104), respectively, determine the well-known collective transverse and longitudinal plasmon modes [9, 20]. In the long-wavelength limit $(\omega \gg q)$, for example, the explicit solutions are:

$$
T: \omega_{T}^{2}(q)=\omega_{0}^{2}+\frac{6}{5} q^{2}, \quad L: \omega_{L}^{2}(q)=\omega_{0}^{2}+\frac{3}{5} q^{2},
$$

with the plasma frequency $\omega_{0}^{2} \equiv \frac{1}{3} m_{D}^{2}$, cf. Eq. (82), which characterizes an ultrarelativistic neutral plasma. Again this is in agreement with the one-loop calculations of finite temperature field theory $\left(T \gg m_{e}\right)$. We remark that beyond the present collisionless approximation these modes naturally aquire a finite width [20].

We now turn to the case of a fully interacting neutrino-antineutrino beam impinging on an electronpositron plasma. We remind ourselves of the two limiting cases introduced in the preceding section concerning the orientation of the wave vector $\vec{q}$ with respect to the outward normal vector $\vec{n}$, i.e. $\vec{q} \| \vec{n}$ (Case I) and $\vec{q} \perp \vec{n}$ (Case II). In both cases, we concentrate on the interesting possibility that the weak interaction term might become comparable to the purely electromagnetic term $\propto e^{2}$ in Eq. (74). Due to the intrinsic smallness of the weak coupling constant this may happen only, when the neutrino-antineutrino response functions become large, close to the singularities found in Eqs. (89)(92) or in Eqs. (95)-(100). Otherwise the neutrino effects can be treated as small perturbations of previous electron-positron plasma results, as we shall see.

Case I. We observe here that the neutrino-antineutrino response function obtained in Eqs. (89)(92) has the same tensor structure as the electron-positron one of Eqs. 83)-(86). Considering the product of the two appearing in Eq. (74), $\mathcal{C}^{\alpha \beta} \equiv M^{(e) \alpha \gamma} M_{\gamma}^{(\nu) \beta}$, we obtain:

$$
\begin{aligned}
& \mathcal{C}^{00}=\left(1-\frac{\omega^{2}}{q^{2}}\right) M^{(e) 00} M^{(\nu) 00} \\
& \mathcal{C}^{0 i}=\mathcal{C}^{i 0}=\frac{q^{0} q^{i}}{q^{2}} \mathcal{C}^{00}
\end{aligned}
$$




$$
\mathcal{C}^{i j}=-\left(\delta^{i j}-\frac{q^{i} q^{j}}{q^{2}}\right) M_{T}^{(e)} M_{T}^{(\nu)}+\frac{q^{i} q^{j}}{q^{2}} \frac{\omega^{2}}{q^{2}} \mathcal{C}^{00}
$$

with $\omega \equiv\left|q^{0}\right|$ and $q \equiv|\vec{q}|$. Therefore, we may still distinguish transverse $(T)$ and longitudinal $(L)$ current fluctuations which do not mix, similarly to the case of a purely electromagnetic plasma.

In analogy to Eqs. (103) and (104), we thus obtain from Eq. (74) two equations which now determine the neutrino 'beam' electron-positron plasma dispersion relations $(\vec{q} \| \vec{n})$ :

$$
\begin{aligned}
& T: \quad\left(1-\left[\frac{e^{2}}{\left(q^{0}\right)^{2}-q^{2}}+2 c_{V}^{2} G_{F}^{2} M_{T}^{(\nu)}\right] M_{T}^{(e)}\right)^{3}=0, \\
& L: \quad 1-\left[\frac{e^{2}}{q^{2}}+2 c_{V}^{2} G_{F}^{2}\left(1-\frac{\left(q^{0}\right)^{2}}{q^{2}}\right)^{2} M^{(\nu) 00}\right] M^{(e) 00}=0,
\end{aligned}
$$

with $q \equiv|\vec{q}|$.

In the long-wavelength limit $(\omega \gg q)$ and to lowest order in $G_{F}^{2}$ we obtain, for example, from Eq. (109) the equation:

$$
\begin{aligned}
0= & \omega^{2}-q^{2}-\omega_{0}^{2}\left(1+\frac{1}{5}\left(\frac{q}{\omega}\right)^{2}+\ldots\right) \\
& -\omega_{0}^{2} a^{2}\left(\frac{4(1-z)+z\left(1-z^{2}\right)}{36}-\frac{\left(1-z^{2}\right)^{2}}{24} \frac{q}{\omega}+\frac{4(1-z)-3 z\left(1-z^{2}\right)^{2}}{60}\left(\frac{q}{\omega}\right)^{2}+\ldots\right),
\end{aligned}
$$

with $\omega \equiv\left|q^{0}\right|, z \equiv \cos \theta_{\max }$, and where we indicated the neglected higher order terms in $q / \omega$. We introduced the dimensionless constant:

$$
a^{2} \equiv \frac{1}{2 e^{2}} G_{F}^{2} m_{w}^{2} m_{D}^{2}
$$

which governs the strength of the neutrino effects. The solution of Eq. (111) describes the transverse plasmon in a neutrino 'beam' electron-positron plasma.

However, as we anticipated, the smallness of the weak coupling constant makes the influence of the neutrino terms completely negligible here. Considering Type II supernova conditions and setting $T_{e} \approx 1 \mathrm{MeV}$ and $T_{\nu} \approx 10 \mathrm{MeV}$, we find that $a^{2} \approx 10^{-22}$. Omitting the neutrino contribution and solving reproduces the first of Eqs. (105).

A similar analysis, i.e. for $\omega>q$, applies to Eq. (110) which the longitudinal plasmon in a neutrino 'beam' electron-positron plasma. Again the neutrinos have a negligible effect under supernova conditions.

We now consider the dispersion relations implicit in Eqs. (109) and (110) close to the resonance frequency, i.e. $\omega \approx \Omega_{\|} \equiv z q$, which lies in the electron-positron Landau damping regime with $0<\omega<$ $q$, considering $0<z<1$ from now on $\left(0<\theta_{\max }<\pi / 2\right)$. 2

In this case, we expect the frequency $q^{0}$, and correspondingly $\omega$, to aquire a finite imaginary part, instead of the infinitesimal $i \epsilon$ representing the retarded boundary condition [9], cf. Eqs. (68), (69) or (80). Therefore, replacing $q^{0}+i \epsilon \longrightarrow \omega+i \gamma$, the 'Landau logarithms' and imaginary parts of the calculated response functions have to be reconsidered. We rewrite $\omega \equiv z q+\xi$, anticipating that $\xi \ll q z$, and will use:

$$
\ln \frac{\omega+q+i \gamma}{\omega-q+i \gamma}=\ln \frac{1+z}{1-z}-i \pi \operatorname{Sign} \gamma+\ln \left(1+\frac{\xi+i \gamma}{q(1+z)}\right)-\ln \left(1-\frac{\xi+i \gamma}{q(1-z)}\right)
$$

\footnotetext{
${ }^{2}$ For the case of an ultrarelativistic pure electron-positron plasma in equilibrium it can be shown that no solution, for example, of the dispersion equation (104) exists with $0<\omega<q$.
} 
where $\operatorname{Sign} \gamma \equiv \gamma /|\gamma|$. This is most appropriate for small $\xi$ and $\gamma$, reproducing the usual result for $\gamma \rightarrow 0^{+}$.

Specifically, we reconsider Eq. (110) and take only the dominant singular term $\propto\left(z q-q^{0}\right)^{-1}$ in $M^{(\nu) 00}$ into account, cf. Eq. (89). Thus we obtain more explicitly:

$$
1+\left[\frac{m_{D}^{2}}{q^{2}}+a^{2} \frac{1-z}{2}\left(1-\frac{(\omega+i \gamma)^{2}}{q^{2}}\right)^{2} \frac{q+\omega+i \gamma}{z q-\omega-i \gamma}\right]\left[1-\frac{1}{2} \frac{\omega+i \gamma}{q} \ln \frac{\omega+q+i \gamma}{\omega-q+i \gamma}\right]=0,
$$

with $\omega \equiv z q+\xi$. We recall that $a^{2} \ll 1$.

It is easy to see that for a solution with $\omega \approx z q$ the term $\propto a^{2}$ has to behave qualitatively such that (at least) $(\xi, \gamma) / q \sim a^{2} q^{2} / m_{D}^{2} \ll 1$, particularly in the long-wavelength limit with $q^{2} / m_{D}^{2} \ll 1$. Consequently, using Eq. (113), we expand Eq. (114) up to second order in $\xi / q$ or $\gamma / q$. Separating real and imaginary parts, it is straightforward to solve the resulting equations. We obtain:

$$
\begin{aligned}
\frac{\xi}{q} & =\frac{1}{2} a^{2}\left(q / m_{D}\right)^{2}\left(1-z^{2}\right)^{3} \frac{(\pi z / 2)^{2}+f^{2}(z)+f(z)\left(q / m_{D}\right)^{2}}{(\pi z / 2)^{2}+\left[f(z)+\left(q / m_{D}\right)^{2}\right]^{2}} \\
\frac{|\gamma|}{q} & =\frac{\pi}{2} a^{2}\left(q / m_{D}\right)^{4} \frac{\left(1-z^{2}\right)^{3}}{(\pi z / 2)^{2}+\left[f(z)+\left(q / m_{D}\right)^{2}\right]^{2}}
\end{aligned}
$$

neglecting higher order in $a^{2}$ corrections and defining:

$$
f(z) \equiv 1-\frac{1}{2} z \ln \frac{1+z}{1-z} .
$$

These results are consistent with the applied expansions, noting that $\xi \propto G_{F}^{2} / e^{2}$ and $\gamma \propto G_{F}^{2} / e^{4}$, particularly in the long-wavelength limit.

Recalling $\omega \equiv z q+\xi$, we thus obtain a pair of longitudinal pharon modes ('Type I', i.e. for $\vec{q} \| \vec{n}$ ), with the real part of the dispersion relation in the long-wavelength limit given by:

$$
\omega(q)=z q+\frac{1}{4 e^{2}} G_{F}^{2} m_{w}^{2}\left(1-z^{2}\right)^{3} q^{3},
$$

one, a growing and the other, a decaying mode, depending on the sign of $\gamma$.

Analogously, we analyze the transverse dispersion relation to be calculated from Eq. (109) for $\omega<q$. In this case, we find a pair of transverse pharon modes (Type I) with:

$$
\begin{aligned}
\frac{\xi}{q} & =-\frac{1}{4} a^{2}\left(q / m_{D}\right)^{2}\left(1-z^{2}\right)^{3} \frac{(\pi / 2)^{2}\left(1-z^{2}\right)+\left(z^{2} /\left(1-z^{2}\right)\right) g^{2}(z)+2 g(z)\left(q / m_{D}\right)^{2}}{(\pi z / 2)^{2}+\left[\left(z^{2} /\left(1-z^{2}\right)\right) g(z)+2\left(q / m_{D}\right)^{2}\right]^{2}} \\
\frac{|\gamma|}{q} & =\frac{\pi}{4} a^{2}\left(q / m_{D}\right)^{4} \frac{z^{-1}\left(1-z^{2}\right)^{3}}{(\pi z / 2)^{2}+\left[\left(z^{2} /\left(1-z^{2}\right)\right) g(z)+2\left(q / m_{D}\right)^{2}\right]^{2}}
\end{aligned}
$$

and where:

$$
g(z) \equiv 1-\frac{1}{2}\left(z-\frac{1}{z}\right) \ln \frac{1+z}{1-z} .
$$

The corresponding real part of the dispersion relation in the long-wavelength limit is:

$$
\omega(q)=z q-\frac{1}{8 e^{2}} G_{F}^{2} m_{w}^{2}\left(1-z^{2}\right)^{3} q^{3} \frac{(\pi / 2)^{2}\left(1-z^{2}\right)+\left(z^{2} /\left(1-z^{2}\right)\right) g^{2}(z)}{(\pi z / 2)^{2}+\left(z^{2} /\left(1-z^{2}\right)\right)^{2} g^{2}(z)},
$$

with an interesting negative sign in front of the second term. We observe that the transverse pharons are quite sensitive to the geometry parameter $z$.

In particular, in the limit $z \rightarrow 0$, corresponding to a maximally fanned-out 'beam' with $\theta_{\max } \rightarrow \pi / 2$, the 'damping constant' $\gamma(q)$ diverges. In this limit the expansions leading to Eqs. (119) and (120) 
clearly break down. This can be studied in more detail, starting again with Eq. (109) and implementing $q^{0}=\xi+i \gamma$, with $\xi, \gamma \ll q$. However, it leads to a nonpropagating mode with frequency of the same small order of magnitude as the damping constant, which is physically irrelevant to our study.

However, under Type II supernova conditions, with $a^{2} \approx 10^{-22}$, and recalling that we have $1-z^{2}=$ $\sin ^{2} \theta_{\max }=(R / r)^{2}$, in terms of the radius $R$ of the neutrino sphere and the distance $r$ of the electronpositron plasma from its center, a typical value may be $(R / r)^{2} \approx 0.5$ for $R \approx 30 \mathrm{~km}$ [2]. Then, for $q<m_{D}$, the above calculations are accurate and we may roughly estimate, for example, the transverse pharon damping constant, $\gamma \approx 10^{-2} a^{2}\left(q / m_{D}\right)^{4} q$. For a pharon wavelength corresponding to $q \approx m_{D} / 2$ and an electron temperature $T_{e} \approx 1 \mathrm{MeV}$, this yields a growth/decay length (one e-folding) on the order of $10^{9}-10^{11} \mathrm{~km}$. A one-percent increase of the collective mode amplitude squared, i.e. of its energy, means it would have to run through more than $10^{6} \mathrm{~km}$ of plasma, which is simply not there. The longitudinal mode behaves similarly.

Clearly, the above estimates are crude and could be improved by folding the results with the appropriate distributions, depending on the distance from the supernova core (and time). However, in view of the intrinsic weakness of the instabilities, we conclude that it is unlikely that long-wavelength Type I $(\vec{q} \| \vec{n})$ pharon modes play an important role in the outward energy transport processes in Type II supernovae.

Considering the strong momentum dependence of the calculated damping constants, Eqs. (116) and $(120)$, however, the question is raised, whether, at shorter wavelengths, corresponding collective modes could become important instead. As we will discuss in the following subsection in more detail, in this limit, the presently employed semiclassical transport theory breaks down, necessitating further study.

Case II. We recall that here we have $\vec{q} \perp \vec{n}$ and proceed as before. However, the product of the two response matrices appearing in Eq. (74), $\mathcal{C}^{\alpha \beta} \equiv M^{(e) \alpha \gamma} M_{\gamma}^{(\nu) \beta}$, has to be recalculated. Taking the different tensor structure of $M^{(\nu)}$, according to Eqs. (95)-100), into account, we obtain:

$$
\begin{aligned}
\mathcal{C}^{00}= & \left(1-\frac{\left(q^{0}\right)^{2}}{q^{2}}\right) M^{(e) 00} M^{(\nu) 00} \\
\mathcal{C}^{0 i}= & \frac{q^{0} q^{i}}{q^{2}} \mathcal{C}^{00}+n^{i}\left(\frac{q}{q^{0}}-\frac{q^{0}}{q}\right) M^{(e) 00} M_{3}^{(\nu)} \\
\mathcal{C}^{i 0}= & \frac{q^{0} q^{i}}{q^{2}} \mathcal{C}^{00}-\frac{q n^{i}}{q^{0}} M_{T}^{(e)} M_{3}^{(\nu)} \\
\mathcal{C}^{i j}= & \frac{q^{i} q^{j}}{q^{2}} \frac{\left(q^{0}\right)^{2}}{q^{2}} \mathcal{C}^{00}+\frac{q^{i} n^{j}}{q}\left(1-\frac{\left(q^{0}\right)^{2}}{q^{2}}\right) M^{(e) 00} M_{3}^{(\nu)} \\
& -\left(\left(\delta^{i j}-n^{i} n^{j}-\frac{q^{i} q^{j}}{q^{2}}\right) M_{T}^{(\nu)}+n^{i} n^{j} M_{L 1}^{(\nu)}+\frac{n^{i} q^{j}}{q} M_{3}^{(\nu)}\right) M_{T}^{(e)}
\end{aligned}
$$

with $q \equiv|\vec{q}|$. We observe that $\mathcal{C}^{\mu \nu} \neq \mathcal{C}^{\nu \mu}$.

In the present case, we consider again two different kinds of current fluctuations, when evaluating Eq. (74): $\delta \vec{J}(e)(q) \perp \vec{q}, \vec{n}$ ('Out'), i.e. fluctuations which are perpendicular to the plane spanned by $\vec{q}$ and $\vec{n}$, and fluctuations with $\delta \vec{J}^{(e)}(q)$ in this plane ('In'). Thus we obtain the following two equations which determine the neutrino 'beam' electron-positron plasma dispersion relations $(\vec{q} \perp \vec{n})$ :

$$
\begin{aligned}
& \text { Out : }\left(1-\left[\frac{e^{2}}{\left(q^{0}\right)^{2}-q^{2}}+2 c_{V}^{2} G_{F}^{2} M_{T}^{(\nu)}\right] M_{T}^{(e)}\right)^{3}=0, \\
& \text { In }: \quad\left(1-2 c_{V}^{2} G_{F}^{2} M_{L 1}^{(\nu)} M_{T}^{(e)}\right)\left(1-\left[\frac{e^{2}}{q^{2}}+2 c_{V}^{2} G_{F}^{2}\left(1-\frac{\left(q^{0}\right)^{2}}{q^{2}}\right)^{2} M^{(\nu) 00}\right] M^{(e) 00}\right)
\end{aligned}
$$




$$
+\left(2 c_{V}^{2} G_{F}^{2}\right)^{2}\left(2-\frac{q^{2}}{\left(q^{0}\right)^{2}}-\frac{\left(q^{0}\right)^{2}}{q^{2}}\right)\left(M_{3}^{(\nu)}\right)^{2} M_{T}^{(e)} M^{(e) 00}=0
$$

with $q^{0} \equiv \omega+i \gamma$. We observe that Eq. (127) has the same formal structure as Eq. (109) before. Furthermore, if we set $G_{F}^{2}$ to zero, these equations reproduce the transverse and longitudinal electronpositron plasmon dispersion equations (103) and (104).

Guided by our analysis of the transverse plasmon dispersion relation under neutrino flux for $\vec{q} \| \vec{n}$, Eqs. (109)-(112), we expect only a negligible perturbative influence of the neutrino interactions in Eq. (127), since they are again suppressed by the factor $a^{2} \approx 10^{-22}$. In particular, the present structure of $M_{T}^{(\nu)}$ is a smoothly deformed version of $M_{T}^{(e)}$, compare Eqs. (86) and (97), with no additional singularity. Thus, the corresponding collective mode shows no particularly interesting behavior and presents a second kind of perturbatively deformed transverse plasmon.

Finally, we consider Eq. (128), describing a geometry which resembles the one where two-stream instabilities arise in other plasmas [8, 9, 10]. We attempt to find pharon type solutions in the present case as well. For this purpose, we take into account the leading root-singular terms, which contribute here from Eqs. (95), (98), and (100). The singularities occur at the resonance frequencies $\omega=\Omega_{\perp}^{ \pm} \equiv$ $\pm q s$, as $q_{z}^{2} \equiv\left(q^{0}\right)^{2}-q^{2}\left(1-z^{2}\right) \rightarrow 0$, where $q \equiv|\vec{q}|$ and $z \equiv \cos \theta_{\max }$.

We concentrate on the positive frequency solutions of the dispersion equation (128) and are particularly interested in those with a positive imaginary part, which grow exponentially in time. Therefore, we consider $q^{0} \equiv \omega+i \gamma$, with $\omega \approx q s>0$, defining $s \equiv \sin \theta_{\max }$. Then, Eq. (128) assumes a slightly simpler form:

$$
0=1+m_{q}^{2} \tilde{M}^{(e) 00}+a^{2} \frac{z}{2} \frac{q^{0}}{\sqrt{q_{z}^{2}}}\left[\frac{\left(q^{0}\right)^{2}}{q^{2}}-1\right]\left(\left[\frac{\left(q^{0}\right)^{2}}{q^{2}}-1\right] \tilde{M}^{(e) 00}+\frac{1}{2} \tilde{M}_{T}^{(e)}\left[1+m_{q}^{2} \tilde{M}^{(e) 00}\right]\right)+O\left(a^{4}\right)
$$

with the dimensionless effective coupling constant $a^{2}$, Eq. (112). The terms of $O\left(a^{4}\right)$ will be neglected in the following, since their singularities cancel. Furthermore, we conveniently define:

$$
m_{q}^{2} \equiv m_{D}^{2} / q^{2}, \quad \tilde{M}^{(e) 00} \equiv-e^{2} M^{(e) 00} / m_{D}^{2}, \quad \tilde{M}_{T}^{(e)} \equiv 2 e^{2} M_{T}^{(e)} / m_{D}^{2}
$$

cf. Eqs. (83) and (86). Recalling the smallness of $a^{2}$, it is obvious that any interesting solution must arise close the resonance frequency $\Omega_{\perp}^{+}=q s(0<s<1)$.

Setting $\omega \equiv q s+\xi$ and assuming $\xi, \gamma \ll q s$, it is useful to expand Eq. (129) in terms of the small complex quantity $\kappa \equiv(\xi+i \gamma) /(2 q s)$. Here we make use of Eq. (113) once more, in order to expand the Landau logarithms. Then, expanding to leading order in $\kappa$, it is straightforward to arrive at the 'formal solution':

$$
\xi+i \gamma=q a^{4} \frac{s\left(1-s^{2}\right)^{3}}{128\left[\left(1+m_{q}^{2} f(s)\right)^{2}+(\pi s / 2)^{2}\right]^{2}}\left(h(s)\left[1+m_{q}^{2}(f(s)-(i \pi s / 2) \operatorname{Sign} \gamma)\right]\right)^{2},
$$

where:

$$
\begin{aligned}
h(s) \equiv & 2 s^{2} g(s)+i \pi s\left(s^{2}-1\right) \operatorname{Sign} \gamma+4\left(s^{2}-1\right)(f(s)+(i \pi s / 2) \operatorname{Sign} \gamma) \\
& +m_{q}^{2}(f(s)+(i \pi s / 2) \operatorname{Sign} \gamma)\left(2 s^{2} g(s)+i \pi s\left(s^{2}-1\right) \operatorname{Sign} \gamma\right) .
\end{aligned}
$$

The functions $f$ and $g$ were defined in Eqs. (117) and (121). The appearance of Sign $\gamma$ on the right-hand side restricts the possibility of an explicit solution.

After some algebra, one obtains a criterion for a solution to exist in the relevant regime $(0<s<1)$ :

$$
\left(-1+s^{2}\left[1+m_{q}^{2} g(s) / 2\right]\right)\left(\pi^{2} m_{q}^{2} s^{2}+4 f(s)\left[1+m_{q}^{2} f(s)\right]\right)+2 s^{2} g(s)\left[1+m_{q}^{2} f(s)\right]<0,
$$


which in the long-wavelength limit $\left(m_{q}^{2}>1\right)$ can only be fullfilled for sufficiently small $s$, i.e. sufficiently small opening angle of the neutrino momentum distribution. Clearly, taking only the leading terms in this limit into account, no solution exists. On the other hand, for $m_{D} / q=2$, for example, the solvability criterion requires $s<0.426$, corresponding to $\theta_{\text {max }} \approx 25^{\circ}$.

It is obvious, however, from Eq. (131) that any pharon ('TypeII', i.e. for $\vec{q} \perp \vec{n}$ ) solution here will have $\xi, \gamma \propto a^{4} \propto G_{F}^{4}$, with no particular factors especially enhancing the damping constant. We refrain from giving explicitly the not very illuminating lengthy expressions.

Instead, we conclude that in the supernova environment the growth rate of the presently studied Type II pharons is suppressed by an extra factor of $a^{2} \approx 10^{-22}$, as compared to Type I. Consequently, these modes do not contribute at all in this case.

\subsubsection{Discussion}

The detailed calculations in the previous subsections are based on the stationary electron and neutrino distribution functions, $f_{S}^{(e)}(k)$ and $f_{S}^{(\nu)}(k)$ of Eqs. $(75)$ and $(76)$, respectively. While $f_{S}^{(e)}(k)$ is adequate for the supernova scenario discussed here, the question arises as to whether the sharp azimuthal angle cut-off, present in $f_{S}^{(\nu)}(k)$, may not cause spurious effects or invalidate our semiclassical transport approach.

In fact, the semiclassical approximation of Section 2.2 is based on the expansion of the full quantum transport equations in powers of $\hbar$, appearing especially in the dimensionless combination $\hbar \partial_{x} \cdot \partial_{p}$, cf. Eqs. (6) and (20)-(22) in Section 2.1. Therefore, a cut-off on a spacelike momentum coordinate, corresponding to the angle $\theta_{\max }$ between three-momentum and outward normal direction, may produce large higher order corrections. These are controlled, however, in the long-wavelength limit. We recall that in the derivation of the linear response theory in Section 3.1, the space-time gradients $\partial / \partial x^{\mu}$ become the four-momenta $q_{\mu}$, beginning with Eq. (65). In the long-wavelength limit, it is generally required that $q$, which probes the spatial inhomogeneity of the (stationary) system, be small compared to the relevant momentum (gradient) scales, i.e. the temperatures $T_{e}, T_{\nu}$. Otherwise, the response functions, see Eq. (80), would inherit neglected higher order terms in $q^{\mu} \partial_{k}^{\nu}$, which correspond to going from Eq. (6) to Eq. (46).

A truly microscopic transport calculation of the neutrino distribution, as they are released from the neutrino sphere, is an interesting topic for future work [1, 2]. We wish to conclude by illustrating the modifications resulting from a more realistic smooth cut-off neutrino momentum distribution.

For example, we consider the azimuthal angle integral which contributes the singular term $\propto$ $\left(q+q^{0}\right) /\left(z q-q^{0}\right)$ to the neutrino response function $M^{(\nu) 00}$, Eq. (89), which in turn is essential for the longitudinal Type I pharon originating in Eq. (110). Following the radial momentum and polar angle integrations, one encounters the integral:

$$
I \equiv q^{2} \int_{-1}^{1} d z\left(q^{0}+i \epsilon-|\vec{q}| z\right)^{-2} F(z)
$$

where we replaced the previous sharp cut-off, i.e. $\Theta\left(z-z_{m}\right)$, by the smooth function $F$,

$$
F(z) \equiv N_{+}^{-1}\left(\arctan \left[\sqrt{\alpha}\left(z-z_{m}\right)\right]+\arctan \left[\sqrt{\alpha}\left(1+z_{m}\right)\right]\right),
$$

where $z_{m} \equiv \cos \theta_{\max }$, and we have introduced the convenient abbreviations:

$$
N_{ \pm} \equiv \arctan \left[\sqrt{\alpha}\left(1-z_{m}\right)\right] \pm \arctan \left[\sqrt{\alpha}\left(1+z_{m}\right)\right] .
$$

Thus, we have $F(-1)=0, F(1)=1$ and the Lorentzian derivative,

$$
F^{\prime}(z)=\frac{\sqrt{\alpha} N_{+}^{-1}}{1+\alpha\left(z-z_{m}\right)^{2}} .
$$


The parameter $\alpha>0$ determines the steepness of the sigmoid cut-off, which ultimately should be related to the emission characteristics of the neutrino sphere [2].

Then, after a partial integration, we obtain an integral with three complex simple poles,

$$
I=\frac{q^{0}+|\vec{q}|}{|\vec{q}|}+\frac{q^{2}}{|\vec{q}|^{2}} \int_{-1}^{1} d z \frac{F^{\prime}(z)}{z-\left(q^{0}+i \epsilon\right) /|\vec{q}|}
$$

which can be solved analytically. The final result is:

$$
\begin{aligned}
I= & 1+\frac{q^{0}}{|\vec{q}|}+\frac{q^{2}\left(\sqrt{\alpha} N_{+}\right)^{-1}}{\left(q^{0}-|\vec{q}| z_{m}\right)^{2}+|\vec{q}|^{2} / \alpha}\left[\sqrt{\alpha} N_{+}\left(z_{m}-\frac{q^{0}}{|\vec{q}|}\right)+\ln \frac{q^{0}-|\vec{q}|}{q^{0}+|\vec{q}|}+\frac{1}{2} \ln \frac{1+\alpha\left(1+z_{m}\right)^{2}}{1+\alpha\left(1-z_{m}\right)^{2}}\right] \\
& \stackrel{a \rightarrow \infty}{\longrightarrow}\left(1-z_{m}\right) \frac{q^{0}+|\vec{q}|}{q^{0}-|\vec{q}| z_{m}} .
\end{aligned}
$$

Thus the previous result is recovered in the sharp cut-off limit.

We observe that the previous resonance pole at $q^{0}=\Omega_{\|} \equiv|\vec{q}| z_{m}$ is moved symmetrically off the real axis. However, closer inspection shows that the above result is not singular any more as $q^{0} \rightarrow|\vec{q}|\left(z_{m} \pm i / \sqrt{\alpha}\right)$. We recalculated the complete $M^{(\nu) 00}$-component of the neutrino response function with the smooth cut-off function $F(z)$ and found a corresponding result.

Therefore, in accordance with intuitive expectation, a physically more appropriate moderate smoothing of the angular dependence of the neutrino momentum distribution turns the pharon resonance poles into resonances with a finite width, which decreases with increasing sharpness of the cut-off (i.e. as $\alpha \rightarrow \infty$ ). Most likely, this implies that realistic growth rates for the unstable modes will be lower than estimated optimistically in Section 3.2.3 .

\section{Conclusions}

In this work, we studied the collective modes which were earlier conjectured to produce anomalously large instabilities in the course of the interaction of the neutrino 'beam' with the plasma sphere in Type II supernovae [5].

For this purpose, we derived the semiclassical transport theory based on the Dirac field equations for neutrinos and electrons, which are coupled according to the Standard Model. Our results also allow for the handling of situations with strong spin-polarizing magnetic fields, which we did not consider here. We derived a related linear response theory and applied it in a detailed supernova scenario, adapted from Bethe's review [2].

We studied, in particular, the modifications of the usual transverse and longitudinal plasmons of an electron-positron plasma, which are caused by a high-power beam-like flux of neutrinos, such as the almost radially outward streaming neutrinos, which are released from the neutrino sphere surrounding the supernova core. In the collisionless approximation, we found only a very weak perturbative effect and no induced imaginary part of the dispersion relation. This is due to the suppression of all neutrino effects by the small dimensionless effective coupling constant,

$$
a^{2} \equiv \frac{1}{2 e^{2}} G_{F}^{2} m_{w}^{2} m_{D}^{2} \approx 10^{-22}
$$

under typical Type II supernova conditions.

However, we found new types of growing, as well as damped collective modes, the pharons, which are characterized by an essentially linear dispersion relation $\omega(q) / q \approx$ const in the long-wavelength limit. Their characteristic properties depend on the relative orientation of the neutrino beam, collective mode propagation and electric current fluctuation directions (the Cases I and II are studied in 
Sections 3.2.2 and 3.2.3). They partially overcome the discussed suppression, since a resonance pole arises in the dispersion equations in the region with $\omega(q)<q$. In this region, one finds in an ordinary electron-positron plasma strongly Landau damped modes or, in the ultrarelativistic limit, no (plasmon) modes at all.

As we estimated roughly in Section 3.2.3, although they partially overcome the discussed suppression, the Type I pharon growth rates are still about four, and likely more, orders of magnitude too small to make an impact on supernova evolution. The Type II pharon growth rate is even more suppressed; it is proportional to $G_{F}^{4}$, like a purely weak interaction cross section. Clearly, we have seen that for the electro-weak interaction to be relevant, the effective particle densities have to be sufficiently high, since one has to overcome the suppression expressed by the smallness of $a^{2}$, given above, or its equivalent for more massive particles. We remark that changing the neutrino and electron temperatures, as compared to the typical supernova case discussed after Eq. (122), for example, while keeping the 'wavelength' $m_{D} / q$ fixed, the Type I pharon damping constants grow and the corresponding e-folding lengths drop $\propto T_{\nu}^{-2} T_{e}^{-3}$.

At this point it is worthwhile remarking that more realistic calculations also have to take the collisonal damping into account. This can be incorporated in our approach in the relaxation time approximation in future applications [9].

Finally, we point out that pharon type modes should occur in other situations with a currentcurrent type interaction under two-stream conditions. The anisotropic momentum distribution characteristic of a 'beam' with limited (momentum) opening angle appears to cause the resonance effect exciting these modes by impact on an isotropic plasma. - We did not study here a spatially limited beam or jet, which causes quite different 'hydrodynamic' instabilities.

Pharon modes may perhaps be fed effectively by the still unknown central engine powering gamma ray bursts, for example, see Refs. [21]. There is growing evidence for even truly jet-like processes in the gamma ray burst phenomenon. Furthermore, allowing for other than electro-weak interactions, such modes possibly come into play in the ultimate evaporation of primordial black holes [22].

To summarize, we conclude that the intrinsic weakness of the neutrino caused collective effects, related to the large asymmetry of the electromagnetic and weak coupling strengths, makes it rather unlikely that they play a role in the neutrino energy deposition in the supernova plasma sphere. However, the new pharon type instabilities may be quite relevant in two-stream situations occuring in other astrophysical systems.

\section{Acknowledgements}

H.T.E wishes to thank U. Heinz, St. Mrówczyński, and L. O. Silva for correspondence. - H.T.E and T.K. were supported in part by PRONEX (No. 41.96.0886.00), R.O. would like to thank PRONEX/ FINEP (No. 41.96.0908.00) for partial support, and all three of us acknowledge partial support by CNPq-Brasil.

\section{Appendix}

Here we consider in more detail the structure of the neutrino-antineutrino spinor Wigner function. The general results of Eqs. (7)-(12) can be further specialized for the case of the Standard Model $\nu_{L} \bar{\nu}_{R}$-system in the massless limit. - We follow the notation of Ref. [23] in this Appendix.

In the main part of the paper we use the Dirac-Pauli representation of the $\gamma$-matrices, which are defined by the anticommutation relations $\left\{\gamma^{\mu}, \gamma^{\nu}\right\}=2 g^{\mu \nu}$, i.e. explicitly:

$$
\gamma_{D}^{0}=\left(\begin{array}{cc}
1 & 0 \\
0 & -1
\end{array}\right), \quad \gamma_{D}^{i}=\left(\begin{array}{cc}
0 & \sigma_{i} \\
-\sigma^{i} & 0
\end{array}\right) ; \quad \gamma_{D}^{5}=\left(\begin{array}{cc}
0 & 1 \\
1 & 0
\end{array}\right)
$$


where all entries are $2 \times 2$-matrices themselves; in particular, $\sigma^{i}, i=1,2,3$ denote the standard Pauli matrices. We listed also the chirality operator $\gamma^{5} \equiv i \gamma^{0} \gamma^{1} \gamma^{2} \gamma^{3}$, which anticommutes with all $\gamma^{\mu}$. In the following we will conveniently begin with the chiral Weyl representation:

$$
\gamma_{W}^{0}=\left(\begin{array}{cc}
0 & 1 \\
1 & 0
\end{array}\right) \quad, \quad \gamma_{W}^{i}=\left(\begin{array}{cc}
0 & \sigma_{i} \\
-\sigma^{i} & 0
\end{array}\right) \quad ; \quad \gamma_{W}^{5}=\left(\begin{array}{cc}
-1 & 0 \\
0 & 1
\end{array}\right)
$$

The subscripts $D, W$ presently serve to distinguish the representations, which are related by:

$$
\gamma_{D}^{\mu}=U_{W} \gamma_{W}^{\mu} U_{W}^{\dagger}, \quad U_{W} \equiv \frac{1}{\sqrt{ } 2}\left(1-\gamma_{W}^{5} \gamma_{W}^{0}\right)
$$

i.e. a simple unitary transformation.

In the Weyl representation the stationary Dirac equation separates into a pair of two-component Weyl equations:

$$
\begin{aligned}
E \chi(\vec{p}) & =-\vec{\sigma} \cdot \vec{p} \chi(\vec{p}), \\
E \phi(\vec{p}) & =+\vec{\sigma} \cdot \vec{p} \phi(\vec{p}),
\end{aligned}
$$

the first of which describes the physical $\nu_{l} \bar{\nu}_{R}$-system, as we shall see, while the second one is discarded in the Standard Model. Using $\left\{\sigma^{i}, \sigma^{j}\right\}=2 \delta^{i j}$, one verifies that $E^{2}=|\vec{p}|^{2}$, in both cases. Concentrating on Eq. (143), we consider first the positive energy solution with four-momentum $p_{+}^{\mu}=(E>0, \vec{p})$, which obeys $(\hat{p} \equiv \vec{p} /|\vec{p}|)$ :

$$
\vec{\sigma} \cdot \hat{p} \chi_{+}=-\chi_{+} \cdot
$$

It thus describes the negative helicity (left-handed) neutrino, $\nu_{L}$. Conversely, for the negative energy solution with $p_{-}^{\mu}=(E<0,-\vec{p})$, we obtain:

$$
\vec{\sigma} \cdot(-\hat{p}) \chi_{-}=+\chi_{-},
$$

thus describing a negative four-momentum positive helicity (right-handed) neutrino, which is the positive four-momentum right-handed antineutrino, $\bar{\nu}_{R}$.

We conclude that in the Weyl representation the physical $\nu_{L} \bar{\nu}_{R}$-spinor $\psi^{(\nu)}$ is represented by:

$$
\psi_{W}^{(\nu)}=\left(\begin{array}{c}
\chi=a_{+} \chi_{+}+a_{-} \chi_{-} \\
\phi=0
\end{array}\right),
$$

where $\chi$ is written as a suitably normalized superposition of the two-spinors $\chi_{ \pm}$, e.g., considering plane wave states 23]. Next, we calculate the corresponding four-spinor in the Dirac-Pauli representation:

$$
\psi_{D}^{(\nu)}=U_{W} \psi_{W}^{(\nu)}=\frac{1}{\sqrt{ } 2}\left(\begin{array}{c}
\chi \\
-\chi
\end{array}\right)
$$

where we applied the unitary transformation $U$ defined in Eqs. (142).

We proceed to calculate the bilinear covariants which are needed in Eqs. (7)-(12). Using the explicit form of the $\gamma$-matrices in the Dirac-Pauli representation, Eqs. (140), we obtain:

$$
\bar{\psi}^{(\nu)} \psi^{(\nu)}=0=\bar{\psi}^{(\nu)} \gamma^{5} \psi^{(\nu)}
$$

with $\bar{\psi}^{(\nu)} \equiv \psi^{(\nu) \dagger} \gamma^{0}=\left(\chi^{\dagger} \chi^{\dagger}\right) / \sqrt{ } 2$, dropping the subscript $D$ from now on. Furthermore:

$$
\begin{aligned}
& \bar{\psi}^{(\nu)} \gamma^{0} \psi^{(\nu)}=\chi^{\dagger} \chi=\bar{\psi}^{(\nu)} \gamma^{5} \gamma^{0} \psi^{(\nu)}, \\
& \bar{\psi}^{(\nu)} \gamma^{i} \psi^{(\nu)}=-\chi^{\dagger} \sigma^{i} \chi=\bar{\psi}^{(\nu)} \gamma^{5} \gamma^{i} \psi^{(\nu)} .
\end{aligned}
$$


Finally, using $\sigma^{\mu \nu} \equiv i\left[\gamma^{\mu}, \gamma^{\nu}\right] / 2$, we also obtain:

$$
\bar{\psi}^{(\nu)} \sigma^{\mu \nu} \psi^{(\nu)}=0
$$

Summarizing, only the vector and axial vector (two-point) densities are nonzero for the $\nu_{L} \bar{\nu}_{R^{-}}$ system and, in fact, they are equal. This yields for the neutrino Wigner function components:

$\mathcal{V}_{\mu}^{(\nu)}(x, p)=\mathcal{A}_{\mu}^{(\nu)}(x, p)$, while all other components vanish, see Eqs. (7)-(12).

Presently we made use only of the algebraic properties of the neutrino spinors. It did not enter that the amplitudes $a_{ \pm}$from Eq. (147) actually are to be considered as creation/annihilation operators.

\section{References}

[1] J. Cooperstein, Phys. Rep. 163 (1988) 95.

[2] H. A. Bethe, Rev. Mod. Phys. 62 (1990) 801.

[3] S. Bludman, Da Hsuan Feng, Th. Gaisser and S. Pittel (eds.), Phys. Rep. 256 (1995) 1.

[4] H. A. Bethe, Phys. Rev. Lett. 56 (1986) 1305.

[5] R. Bingham, J. M. Dawson, J. J. Su and H. A. Bethe, Phys. Lett. A193 (1994) 279; J. T. Mendonça, R. Bingham, P.K.Shukla, J. M. Dawson and V.N. Tsytovich, Phys. Lett. A209 (1995) 78; R. Bingham, H. A. Bethe, J.M.Dawson, P.K. Shukla and J. J.Su, Phys. Lett. A220 (1996) 107; P. K. Shukla, R. Bingham, J. T. Mendonca and L. Stenflo, Phys. of Plasmas 5 (1998) 2815; P. K. Shukla, L. Stenflo, R. Bingham, J. T. Mendonca and J. M. Dawson, Physica Scripta T75 (1998) 58; R. Bingham, R. A. Cairns, J. M. Dawson, R. O. Dendy, C. N. Lashmore-Davis, J. T. Mendonca, P. K. Shukla, L. O. Silva and L. Stenflo, Physica Scripta T75 (1998) 61; V. N. Tsytovich, R. Bingham, J. M. Dawson and H. A. Bethe, Astroparticle Physics 8 (1998) 297; P. K. Shukla, L. O. Silva, H. A. Bethe, R. Bingham, J. M. Dawson, L. Stenflo, J. T. Mendonca and S. Dalhed, Plasma Phys. Control. Fusion 41 (1999) A699; L. O. Silva, R. Bingham, J. M. Dawson and W. B. Mori, Phys. Rev. E59 (1999) 2273; L. O. Silva, R. Bingham, J. M. Dawson, P. K. Shukla, N. L. Tsintsadze and J.T. Mendonca, Phys. Rev. D60 (1999) 068701; L. O. Silva, R. Bingham, J. M. Dawson, J. T. Mendonca and P. K. Shukla, Phys. Rev. Lett. 83 (1999) 2703.

[6] L. Bento, Phys. Rev. D61 (2000) 013004.

[7] J.F. Nieves, Electromagnetic properties of a neutrino stream (2000) hep-ph/0001067.

[8] E. S. Weibel, Phys. Rev. Lett. 2 (1959) 83.

[9] E. M. Lifshitz and L. P. Pitaevskii, Physical Kinetics (Pergamon Press, New York, 1981).

[10] St. Mrówczyński, Phys. Lett. B393 (1997) 26.

[11] D. Vasak, M. Gyulassy and H.-Th. Elze, Ann. Phys. (N.Y.) 173 (1987) 462.

[12] H.-Th. Elze, M. Gyulassy and D. Vasak, Nucl. Phys. B276 (1986) 706; Phys. Lett. B177 (1986) 402; H.-Th. Elze and U. Heinz, Phys. Rep. 183 (1989) 81; H.-Th. Elze, Z. Phys. C38 (1988) 211; Z. Phys. C47 (1990) 647.

[13] H.-Th. Elze, M. Gyulassy, D. Vasak, H. Heinz, H. Stöcker and W. Greiner, Mod. Phys. Lett. A2 (1987) 451.

[14] E. Roulet, Neutrinos in physics and astrophysics (1999) hep-ph/9910383. 
[15] P. K. Shukla and L. Stenflo, Phys. Rev. E57 (1998) 2479.

[16] A. V. Kuznetsov and N. V. Mikheev, Plasma influence on neutrino-electron processes in a strong magnetic field (1999) hep-ph/9907493.

[17] A. J. Brizard, H. Murayama and J.S. Wurtele, Phys. Rev. E61 (2000) 4410.

[18] H.-Th. Elze, W. Greiner and J. Rafelski, J. Phys. G6 (1980) L149; G. Schmidt, H.-Th. Elze, J. Rafelski, Nucl. Phys. A364 (1981) 527.

[19] U. Heinz, Phys. Lett. 109A (1985) 385.

[20] J. I. Kapusta, Finite-Temperature Field Theory (Cambridge University Press, Cambridge, 1989).

[21] T. Piran, Phys. Rep. 314 (1999) 575; R. Sari, T. Piran and J. Halpern, Astrophys. J. Lett. 524 (1999) L43; P. Meszaros, M. J. Rees and R. A. M. J. Wijers, New Astron. 4 (1999) 303; B. Paczynski, Gamma-Ray Burst - Supernova Relation (1999) astro-ph/9909048.

[22] J. Kapusta, The last eight minutes of a primordial black hole (1999) astro-ph/9911309.

[23] F. Halzen and A. D. Martin, Quarks and Leptons (John Wiley \& Sons, New York, 1984). 